\title{
Continuous adjoint complement to the Blasius equation
}

Cite as: Phys. Fluids 33, 033608 (2021); https://doi.org/10.1063/5.0037779

Submitted: 17 November 2020 • Accepted: 07 January 2021 • Published Online: 15 March 2021

(iD) Niklas Kühl, (D) Peter Marvin Müller and (iD) Thomas Rung

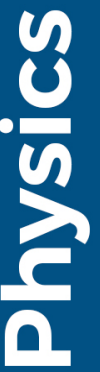

\section{ARTICLES YOU MAY BE INTERESTED IN}

Referee acknowledgment for 2020

Physics of Fluids 33, 020201 (2021); https://doi.org/10.1063/5.0043282

Energy budget analysis and neutral curve characteristics for the linear instability of CouettePoiseuille flow

Physics of Fluids 33, 034102 (2021); https://doi.org/10.1063/5.0033474

The theoretical prediction of the boundary-layer-blockage and external flow choking at moving aircraft in ground effects

Physics of Fluids 33, 036108 (2021); https://doi.org/10.1063/5.0040440

\section{LEARN MORE}

$\underset{\text { Publishing }}{\mathrm{Alp}}$ Author Services

Maximize your publication potential with

English language editing and

translation services

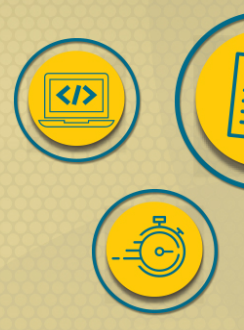

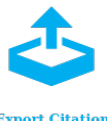

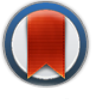

CrossMark 


\title{
Continuous adjoint complement to the Blasius equation
}

\author{
Cite as: Phys. Fluids 33, 033608 (2021); doi: 10.1063/5.0037779 \\ Submitted: 17 November 2020 - Accepted: 7 January 2021 . \\ Published Online: 15 March 2021
}

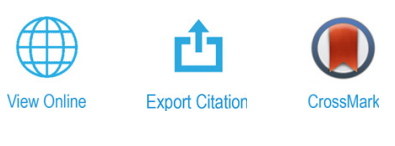

\author{
Niklas Kühl, ${ }^{\text {a) }}$ (D) Peter Marvin Müller, (D) and Thomas Rung (D)
}

\begin{abstract}
AFFILIATIONS
Institute for Fluid Dynamics and Ship Theory, Hamburg University of Technology, Am Schwarzenberg-Campus 4, D-21075 Hamburg, Germany
\end{abstract}

\section{a) Author to whom correspondence should be addressed: niklas.kuehl@tuhh.de}

\begin{abstract}
This manuscript is concerned with a continuous adjoint complement to two-dimensional, incompressible, first-order boundary-layer equations for a flat plate boundary layer. The text is structured into three parts. The first part demonstrates that the adjoint complement can be derived in two ways, following either a first simplify then derive or a first derive and then simplify strategy. The simplification step comprises the classical boundary-layer (BL) approximation, and the derivation step transfers the primal flow equation into a companion adjoint equation. The second part of the paper comprises the analyses of the coupled primal/adjoint BL framework. This leads to similarity parameters, which turn the partial-differential-equation (PDE) problem into a boundary value problem described by a set of ordinarydifferential-equations (ODEs) and support the formulation of an adjoint complement to the classical Blasius equation. Opposite to the primal Blasius equation, its adjoint complement consists of two ODEs, which can be simplified depending on the treatment of advection. It is shown that the advective fluxes, which are frequently debated in the literature, vanish for the investigated self-similar BL flows. Differences between the primal and the adjoint Blasius framework are discussed against numerical solutions, and analytical expressions are derived for the adjoint BL thickness, wall shear stress, and subordinated skin friction and drag coefficients. The analysis also provides an analytical expression for the shape sensitivity to shear driven drag objectives. The third part assesses the predictive agreement between the different Blasius solutions and numerical results for Navier-Stokes simulations of a flat plate BL at Reynolds numbers between $10^{3} \leq \mathrm{Re}_{\mathrm{L}} \leq 10^{5}$. It is seen that the reversal of the inlet and outlet locations and the direction of the flow, inherent to the adjoint formulation of convective kinematics, poses a challenge when investigating real finite length (finite Re-number) flat plate boundary layer problems. Efforts to bypass related issues are discussed.
\end{abstract}

(C) 2021 Author(s). All article content, except where otherwise noted, is licensed under a Creative Commons Attribution (CC BY) license (http:// creativecommons.org/licenses/by/4.0/). https://doi.org/10.1063/5.0037779

\section{INTRODUCTION}

The goal of an adjoint analysis is commonly the efficient computation of derivative information of an integral objective functional with respect to a general control function. The approach has recently gained increasing popularity in local optimization strategies using computational fluid dynamics (CFD). ${ }^{19-21,31,39}$ In continuous space, the dual or adjoint flow state can be interpreted as a co-state and always follows from the underlying primal partialdifferential-equation (PDE) governed model, which describes the flow physics. The cost function definition typically provides necessary boundary conditions and/or source terms to close the formulation and a sound relation between the objective functional and the control.

The formulation of boundary conditions, an appropriate treatment of advective terms, and the adequate discretization are often not intuitively clear in a PDE-based, continuous adjoint (CA) framework. ${ }^{21,29,30,38,39}$ Therefore, the understanding of adjoint flows, as well as the development of numerical strategies, clearly lags behind the primal progress and has motivated the development of discrete adjoint (DA) approaches using automatic differentiation to synchronize the primal and dual development states (see, e.g., Refs. 13, 14, 27, and 28). The DA approach passes over the adjoint PDE and directly bridges the discrete linearized primal flow into a consistent discrete dual approach, cf. a comprehensive discussion in Giles and Pierces ${ }^{11,12}$ or the lecture series by Vassberg and Jameson. ${ }^{42,43}$ Despite the various merits and drawbacks of the DA vs the CA method, the CA approach is unique for its invaluable contribution to a physical understanding and will, therefore, be the method of choice in the present paper.

We intent to contribute to understanding adjoint wall-bounded shear flows by deriving the adjoint complement to a first-order 
boundary-layer (BL) framework. This paper tries to answer the fundamental question if there is an adjoint complement to the similarity transformation inspired by the Blasius solution for a laminar flat plate boundary layer and examines different paths toward a generalized pri$\mathrm{mal} /$ adjoint similarity transformation. With a view to practical implications, attention is given to the influence of the adjoint advection, frequently labeled adjoint transpose convection (ATC). The ATC term is often debated for the invoked robustness and discretization issues, ${ }^{4,7,21,29,32,38}$ and its appearance in analytical BL formulations is of practical interest.

Starting from the non-linear Navier-Stokes (NS) equations, different routes to derive an adjoint BL framework are conceivable. One could first simplify the primal NS equations using the classical $\mathrm{BL}$ approximation for $R e \rightarrow \infty$ to formulate the BL equations, subsequently linearize the BL equations, and finally derive their adjoint companion. On the contrary, the NS equations could be linearized in an initial step, and the adjoint NS companion can subsequently be derived, which is finally reduced to an adjoint $\mathrm{BL}$ approximation for $R e \rightarrow \infty$. In a sense, the two options remind of the above discussed options for obtaining the discrete adjoint from a derive-and-discretize (CA) or a discretize-and-derive (DA) strategy. Both approaches, i.e., simplify-and-derive and derive-and-simplify, will be performed for the first time in this paper to verify their agreement and thus correctness. A more desirable, compact form of the primal BL equations employs a similarity transformation and reduces the BL PDE system into a single ordinary differential equation (ODE) in line with the Blasius solution. Although the similarity transformation is expected to be generalizable, an adjoint similarity transformation was-to the best of our knowledge-never examined directly. This paper outlines a strategy to derive an adjoint complement to the Blasius equation from a general similarity analysis. Interestingly, the adjoint Blasius equations furnish evidence that the ATC term-despite its formal occurrence-remains identical to zero, a fact that is not obvious from the BL PDEs. We demonstrate that the application of the adjoint Blasius equation to a drag objective functional displays strong similarities to a thermal $\mathrm{BL}$ solution at a negative unit Prandtl number.

Previous adjoint-based investigations into BL type flows can be grouped into investigation with respect to either $\mathrm{BL}$ receptivity, modal sensitivity, ${ }^{8,10}$ or BL stability ${ }^{15,18}$ considering the perturbationbased excitation of Tollmien-Schlichting waves ${ }^{1,2}$ or the linearized OrrSommerfeld equation. ${ }^{6,9}$ Hill $^{16,17}$ was the first who investigated the adjoint Orr-Sommerfeld operator and subsequently extended his study toward the adjoint to a parabolic stability equation. The latter was enhanced by Pralits et al. ${ }^{33}$ to derive an adjoint BL framework for the optimization of disturbance control-which somehow differs to our access into the topic of adjoint wall-bounded flow. A very good overview of adjoint applications to fluid-dynamic stability analysis is given in Luchini and Bottaro. ${ }^{26}$ Unlike the previous research, the present paper derives the complete adjoint set of BL equations and compares them with their primal companion. Hence, instead of transposing a few-although particularly important-primal operators, we offer an extensive adjoint-based insight and thus should sharpen the adjoint $\mathrm{BL}$ understanding. The investigated BL flow is admittedly simple. However, we still consider it of fundamental interest for engineering applications. Although findings are not provably valid for more complex flows, e.g., turbulent BL or separated flows, they might still be indicative for attached boundary layers and other virtually unidirectional shear flows. ${ }^{36,41}$ Of particular interest is the disappearance of the ATC term in the Blasius framework, which corresponds to an often employed heuristic simplification. The issue will also be discussed in Sec. VI, which compares different primal/adjoint NS formulations with the results of a primal/adjoint Blasius framework.

This paper is organized as follows: Sec. II is concerned with the derivation of the adjoint BL equations. Section III presents a generalized similarity transformation to condense the primal/dual PDE systems into an ODE framework. Subsequently, we investigate the adjoint Blasius equation numerically in Sec. IV and analytically in Sec. V. Section VI compares BL results with NS solutions for a zero pressure gradient (ZPG) flat plate flow, which are obtained from numerical simulations at Reynolds numbers between $10^{3} \leq \operatorname{Re}_{\mathrm{L}} \leq 10^{5}$. Section VII provides conclusions and outlines future research. Within the publication, Einstein's summation convention is used for lower-case Latin subscripts. Vectors and tensors are defined with reference to Cartesian coordinates, and dimensionless field quantities are consistently marked with an asterisk.

\section{PRIMAL AND ADJOINT BOUNDARY-LAYER EQUATIONS}

This paper deals with incompressible fluids in a steady state, which form flat-plate momentum boundary layers exposed to zero pressure gradients. More generally, the velocity $v_{\mathrm{i}}$ and pressure $p$ follow from the steady incompressible NS equations

$$
\begin{gathered}
\mathrm{R}_{\mathrm{i}}^{\mathrm{v}}: v_{\mathrm{k}} \frac{\partial v_{\mathrm{i}}}{\partial x_{\mathrm{k}}}+\frac{\partial}{\partial x_{\mathrm{k}}}\left[\frac{p}{\rho} \delta_{\mathrm{ik}}-2 \nu S_{\mathrm{ik}}\right]=0, \\
\mathrm{R}^{\mathrm{p}}:-\frac{\partial v_{\mathrm{k}}}{\partial x_{\mathrm{k}}}=0,
\end{gathered}
$$

where $\rho, \nu, S_{\mathrm{ik}}=1 / 2\left(\partial v_{\mathrm{i}} / \partial x_{\mathrm{k}}+\partial v_{\mathrm{k}} / \partial x_{\mathrm{i}}\right)$, and $\delta_{\mathrm{ik}}$ represent the density, kinematic viscosity, symmetric strain rate tensor, and Kronecker delta, respectively. Boundary conditions are given in Table I.

\begin{tabular}{|c|c|c|c|c|}
\hline Boundary type & $v_{\mathrm{i}}$ & $p$ & $\hat{v}_{\mathrm{i}}$ & $\hat{p}$ \\
\hline Inlet & $v_{\mathrm{i}}=v_{\mathrm{i}}^{\mathrm{in}}$ & $\frac{\partial p}{\partial n}=0$ & $\hat{v}_{\mathrm{i}} n_{\mathrm{i}}=-\rho \frac{\partial j_{\Gamma}}{\partial p}$ & $\frac{\partial \hat{p}}{\partial n}=0$ \\
\hline Outlet & $\frac{\partial v_{\mathrm{i}}}{\partial n}=0$ & $\frac{\partial p}{\partial n}=0$ & $\hat{v}_{\mathrm{i}} n_{\mathrm{i}}=-\rho \frac{\partial j_{\Gamma}}{\partial p}$ & $\hat{p} n_{\mathrm{i}}=v_{\mathrm{k}} \hat{v}_{\mathrm{i}} n_{\mathrm{k}}+2 \mu S_{\mathrm{ik}}+\frac{\partial j_{\Gamma}}{\partial v_{\mathrm{i}}}$ \\
\hline Wall & $v_{\mathrm{i}}=0$ & $\frac{\partial p}{\partial n}=0$ & $\hat{v}_{\mathrm{i}} n_{\mathrm{i}}=-\rho \frac{\partial j_{\Gamma}}{\partial p}$ & $\frac{\partial \hat{p}}{\partial n}=0$ \\
\hline
\end{tabular}

TABLE I. Boundary conditions for the primal and adjoint equations. 
Two-dimensional equations for the momentum boundary layer in the $x_{1}-x_{2}$-plane can be derived in two consecutive steps. First, the governing Eqs. (1) and (2) are non-dimensionalized with the reference quantities given in Table II. An exemplary relationship between a dimensional term, these reference values, and non-dimensional quantities marked with an asterisk reads $v_{2} \partial v_{1} / \partial x_{2}=\left(V_{2} V_{1} / \delta\right)$ $\left(v_{2}^{*} \partial v_{1}^{*} / \partial x_{2}^{*}\right)$. Subsequently, a scaling analysis is performed, where it is assumed that the spatial extent in the streamwise $x_{1}$-direction is significantly larger than the extent in the direction of the wall normal $x_{2}$, viz., $L \gg \delta$. The assumption is in line with a flat plate of semi-infinite length $L(\rightarrow \infty)$ in the $x_{1}$ direction (cf. Fig. 2). Thus, the continuity Eq. (2) reveals $V_{2} \propto V_{1} \delta / L$. The scaling analysis is performed in Appendix A using the reference data from Table II. Here, only the resulting $\mathrm{BL}$ equations are given in residual notation, i.e.,

$$
\begin{gathered}
\mathrm{R}_{1}^{\mathrm{v}, \mathrm{BL}}: v_{1} \frac{\partial v_{1}}{\partial x_{1}}+v_{2} \frac{\partial v_{1}}{\partial x_{2}}+\frac{1}{\rho} \frac{\partial p}{\partial x_{1}}-\nu \frac{\partial^{2} v_{1}}{\partial x_{2}^{2}}=0 \\
\mathrm{R}_{2}^{\mathrm{v}, \mathrm{BL}}: \frac{1}{\rho} \frac{\partial p}{\partial x_{2}}=0 \\
\mathrm{R}^{\mathrm{p}, \mathrm{BL}}:-\left(\frac{\partial v_{1}}{\partial x_{1}}+\frac{\partial v_{2}}{\partial x_{2}}\right)=0 .
\end{gathered}
$$

As noted above, the corresponding adjoint $\mathrm{BL}$ equations can be derived in two ways, following either a derive-and-simplify or a simplifyand-derive strategy. In both cases, the derivation step starts with the definition of a surface [volume] based objective functional $j_{\Gamma}\left[j_{\Omega}\right]$ that allows for the construction of an augmented objective, frequently labeled as Lagrangian $L$. Following the derive-and-simplify route, one obtains

$$
\begin{aligned}
L= & \int_{\Gamma} j_{\Gamma} \mathrm{d} \Gamma+\int_{\Gamma_{\text {in }}} \hat{v}_{\mathrm{i}}\left[v_{\mathrm{i}}-n_{\mathrm{i}} v_{\mathrm{i}}^{\text {in }}\right]+\hat{p} \frac{\partial p}{\partial n} \mathrm{~d} \Gamma \\
& +\int_{\Gamma_{\text {out }}} \hat{v}_{\mathrm{i}}\left[\frac{\partial v_{\mathrm{i}}}{\partial n}\right]+\hat{p} \frac{\partial p}{\partial n} \mathrm{~d} \Gamma+\int_{\Gamma_{\text {wall }}} \hat{v}_{\mathrm{i}} v_{\mathrm{i}}+\hat{p} \frac{\partial p}{\partial n} \mathrm{~d} \Gamma \\
& +\int_{\Omega_{\mathrm{O}}} j_{\Omega} \mathrm{d} \Omega+\int_{\Omega} \hat{v}_{\mathrm{i}} \mathrm{R}_{\mathrm{i}}^{\mathrm{v}}+\hat{p} \mathrm{R}^{\mathrm{p}} \mathrm{d} \Omega,
\end{aligned}
$$

where the second volume integral accounts for the field Eqs. (1) and (2), and the boundary integrals consider the corresponding boundary conditions from Table I, $\Gamma=\Gamma_{\text {in }} \cap \Gamma_{\text {out }} \cap \Gamma_{\text {wall }}$. Note that the habitat of an objective does not necessarily coincide with the complete surface or volume, which is why we introduce an additional subscript, viz., $\Gamma_{\mathrm{O}} \cap \Gamma$ and $\Omega_{\mathrm{O}} \cap \Omega$. The local objectives $j_{\Gamma}\left[j_{\Omega}\right]$ disappear on $\Gamma \backslash \Gamma_{\mathrm{O}}$ [in $\Omega \backslash \Omega_{\mathrm{O}}$ ]. The Lagrangian multipliers $\hat{v}_{\mathrm{i}}$ and $\hat{p}$ are frequently labeled as dual or adjoint velocity and pressure. The dimensions of $\hat{v}_{\mathrm{i}}$ and $\hat{p}$ depend on the underlying objective, e.g., $\left[\hat{v}_{\mathrm{i}}\right]=[J] /\left(\left[\mathrm{R}_{\mathrm{i}}^{\mathrm{v}}\right] \mathrm{m}^{3}\right)$ and $[\hat{p}]=[J] /\left(\left[\mathrm{R}^{\mathrm{p}}\right] \mathrm{m}^{3}\right)$, where $[J]=\left[j_{\Omega}\right] \mathrm{m}^{3}\left([J]=\left[j_{\Gamma}\right] \mathrm{m}^{2}\right)$ represents the units of the integral volume-based (surface-based) objective. First order optimality conditions force a vanishing derivative of the

TABLE II. Reference quantities of the $2 \mathrm{D}$ governing equations.

\begin{tabular}{lccccc}
\hline \hline Primal quantity/operator & $v_{1}$ & $v_{2}$ & $p$ & $\partial \phi / \partial x_{1}$ & $\partial \phi / \partial x_{2}$ \\
Reference value & $V_{1}$ & $V_{2}$ & $P$ & $L$ & $\Delta$ \\
Adjoint quantity/operator & $\hat{v}_{1}$ & $\hat{v}_{2}$ & $\hat{p}$ & $\partial \hat{\phi} / \partial x_{1}$ & $\partial \hat{\phi} / \partial x_{2}$ \\
Reference value & $\hat{V}_{1}$ & $\hat{V}_{2}$ & $\hat{P}$ & $L$ & $\hat{\delta}$ \\
\hline \hline
\end{tabular}

Lagrangian in all dependent directions $\left(\delta_{\mathrm{v}_{\mathrm{i}}} L \cdot \delta v_{\mathrm{i}}=0 \forall \delta v_{\mathrm{i}}\right.$, $\left.\delta_{\mathrm{p}} L \cdot \delta p=0 \forall \delta p\right)$. The related derivatives read

$$
\begin{aligned}
\delta_{\mathrm{v}_{\mathrm{i}}} L \cdot \delta v_{\mathrm{i}}= & \int_{\Gamma_{\mathrm{in}}} \hat{v}_{\mathrm{i}}\left(\delta v_{\mathrm{i}}\right) \mathrm{d} \Gamma+\int_{\Gamma_{\text {out }}} \hat{v}_{\mathrm{i}} \frac{\partial\left(\delta v_{\mathrm{i}}\right)}{\partial n} \mathrm{~d} \Gamma+\int_{\Gamma_{\text {wall }}} \hat{v}_{\mathrm{i}}\left(\delta v_{\mathrm{i}}\right) \mathrm{d} \Gamma \\
& +\int_{\Gamma} \delta v_{\mathrm{i}}\left[\frac{\partial j_{\Gamma}}{\partial v_{\mathrm{i}}}+v_{\mathrm{k}} \hat{v}_{\mathrm{i}} n_{\mathrm{k}}+2 \nu \hat{S}_{\mathrm{ik}} n_{\mathrm{k}}-\hat{p} n_{\mathrm{i}}\right]-\left(\delta S_{\mathrm{ik}}\right) \hat{v}_{\mathrm{i}} \nu \mathrm{d} \Gamma \\
& +\int_{\Omega} \delta v_{\mathrm{i}}\left[\hat{v}_{\mathrm{k}} \frac{\partial v_{\mathrm{k}}}{\partial x_{\mathrm{i}}}-v_{\mathrm{k}} \frac{\partial \hat{v}_{\mathrm{i}}}{\partial x_{\mathrm{k}}}-2 \nu \frac{\partial \hat{S}_{\mathrm{ik}}}{\partial x_{\mathrm{k}}}+\frac{\partial \hat{p}}{\partial x_{\mathrm{i}}}+\frac{\partial j_{\Omega}}{\partial v_{\mathrm{i}}}\right] \mathrm{d} \Omega \\
\stackrel{!}{=} & 0 \forall \delta v_{\mathrm{i}}, \\
\delta_{\mathrm{p}} L \cdot \delta p= & \int_{\Gamma_{\mathrm{in}}} \hat{p} \frac{\partial \delta p}{\partial n} \mathrm{~d} \Gamma+\int_{\Gamma_{\text {out }}} \hat{p} \frac{\partial \delta p}{\partial n} \mathrm{~d} \Gamma+\int_{\Gamma_{\text {wall }}} \hat{p} \frac{\partial \delta p}{\partial n} \mathrm{~d} \Gamma \\
& +\int_{\Gamma} \delta p\left[\frac{\partial j_{\Gamma}}{\partial p}+\hat{v}_{\mathrm{i}} \frac{1}{\rho} n_{\mathrm{i}}\right] \mathrm{d} \Gamma \\
& +\int_{\Omega} \delta p\left[-\frac{\partial \hat{v}_{\mathrm{i}}}{\partial x_{\mathrm{i}}} \frac{1}{\rho}+\frac{\partial j_{\Omega}}{\partial p}\right] \mathrm{d} \Omega \\
! & 0 \forall \delta p .
\end{aligned}
$$

Here, $\hat{S}_{\mathrm{ik}}=1 / 2\left(\partial \hat{v}_{\mathrm{i}} / \partial x_{\mathrm{k}}+\partial \hat{v}_{\mathrm{k}} / \partial x_{\mathrm{i}}\right)$ represents the adjoint strain rate tensor. The adjoint field equations result from the field integrals in Eqs. (7) and (8); hence,

$$
\begin{gathered}
\mathrm{R}_{\mathrm{i}}^{\hat{v}}:-v_{\mathrm{k}} \frac{\partial \hat{v}_{\mathrm{i}}}{\partial x_{\mathrm{k}}}+\hat{v}_{\mathrm{k}} \frac{\partial v_{\mathrm{k}}}{\partial x_{\mathrm{i}}}+\frac{\partial}{\partial x_{\mathrm{k}}}\left[\hat{p} \delta_{\mathrm{ik}}-2 \nu \hat{S}_{\mathrm{ik}}\right]=-\frac{\partial j_{\Omega}}{\partial v_{\mathrm{i}}}, \\
\mathrm{R}^{\hat{p}}:-\frac{1}{\rho} \frac{\partial \hat{v}_{\mathrm{k}}}{\partial x_{\mathrm{k}}}=-\frac{\partial j_{\Omega}}{\partial p} .
\end{gathered}
$$

The corresponding boundary conditions follow from the surface integrals in Eqs. (7) and (8) and are summarized in Table I. They depend on the underlying surface based objective functional $j_{\Gamma}$, and we refer to Refs. 24 and 25 for a more detailed discussion.

Note that an additional cross coupling term, frequently labeled as the ATC, arises in the adjoint system due to the non-linearity of the primal convective momentum transport-e.g., the second term on the left hand side in (9). The term might disappear for compressible flows, cf. Soto and Löhner. ${ }^{38}$ Nevertheless, the ATC terms are also frequently neglected in incompressible formulations due to the related impairment of the numerical robustness. ${ }^{29,39}$ Some authors raise a mathematical argument based on the approximation order, viz., $\delta[\underline{v} \cdot(\underline{\nabla} \underline{v})]$ $=\delta \underline{v} \cdot(\underline{\nabla} \underline{v})+\underline{v} \cdot(\underline{\nabla} \delta \underline{v}) \approx \mathcal{O}(\delta \underline{v})+\mathcal{O}(\underline{\nabla} \delta \underline{v})$, to justify the neglect of the ATC. $4,7,29$ The present manuscript provides another indicator for the physically justifiable neglect of this term.

Similar to the primal problem, the adjoint system (9)-(10) is analyzed with respect to the spatial scale/order of magnitude in a nondimensional setting. The corresponding reference quantities follow from Table II. We define a scaling of the $x_{2}$-derivative of an adjoint quantity with $\hat{\delta}$, viz., $v_{2} \partial \hat{v}_{1} / \partial x_{2}=\left(V_{2} \hat{V}_{1} / \hat{\delta}\right)\left(v_{2}^{*} \partial \hat{v}_{1}^{*} / \partial x_{2}^{*}\right)$. The scaling analysis of Eqs. (9) and (10) is performed in Appendix B for an objective that does not depend on the primal pressure, e.g., $\partial j_{\Omega} / \partial p=0$, and yields

$$
\begin{gathered}
\mathrm{R}_{1}^{\hat{v}, \mathrm{BL}}:-v_{1} \frac{\partial \hat{v}_{1}}{\partial x_{1}}-v_{2} \frac{\partial \hat{v}_{1}}{\partial x_{2}}+\hat{v}_{1} \frac{\partial v_{1}}{\partial x_{1}}+\frac{\partial \hat{p}}{\partial x_{1}}-\nu \frac{\partial^{2} \hat{v}_{1}}{\partial x_{2}^{2}}=-\frac{\partial j_{\Omega}}{\partial v_{1}}, \\
\mathrm{R}_{2}^{\hat{v}, \mathrm{BL}}: \hat{v}_{1} \frac{\partial v_{1}}{\partial x_{2}}+\frac{\partial \hat{p}}{\partial x_{2}}=-\frac{\partial j_{\Omega}}{\partial v_{2}}
\end{gathered}
$$




$$
\mathrm{R}^{\hat{p}, \mathrm{BL}}:-\left(\frac{1}{\rho} \frac{\partial \hat{v}_{1}}{\partial x_{1}}+\frac{1}{\rho} \frac{\partial \hat{v}_{2}}{\partial x_{2}}\right)=0 .
$$

Alternatively, Eqs. (11)-(13) can also be derived in a simplify-andderive approach (cf. Fig. 1). The latter starts with a Lagrangian based on the primal BL equations (3)-(5), viz.,

$$
\begin{aligned}
L= & \int_{\Gamma} j_{\Gamma} \mathrm{d} \Gamma+\int_{\Gamma_{\text {wall }}} \hat{v}_{\mathrm{i}} v_{\mathrm{i}}+\hat{p} \frac{\partial p}{\partial n} \mathrm{~d} \Gamma+\int_{\Omega_{0}} j_{\Omega} \mathrm{d} \Omega \\
& +\int_{\Omega} \hat{v}_{1}\left[v_{1} \frac{\partial v_{1}}{\partial x_{1}}+v_{2} \frac{\partial v_{1}}{\partial x_{2}}+\frac{\partial p}{\partial x_{1}} \frac{1}{\rho}-\nu \frac{\partial^{2} v_{1}}{\partial x_{2}^{2}}\right] \\
& +\hat{v}_{2}\left[\frac{\partial p}{\partial x_{2}} \frac{1}{\rho}\right]+\hat{p}\left[\frac{\partial v_{1}}{\partial x_{1}}+\frac{\partial v_{2}}{\partial x_{2}}\right] \mathrm{d} \Omega .
\end{aligned}
$$

Again, first order optimality conditions force vanishing derivatives of the Lagrangian in all dependent directions $\left[\delta_{\mathrm{v}_{\mathrm{i}}} L \cdot \delta v_{\mathrm{i}}=\delta_{\mathrm{v}_{1}} L \cdot \delta\right.$ $\left.v_{1}+\delta_{\mathrm{v}_{2}} L \cdot \delta v_{2}=0 \forall\left(\delta v_{1}, \delta v_{2}\right), \delta_{\mathrm{p}} L \cdot \delta p=0 \forall \delta p\right]$, i.e.,

$$
\begin{aligned}
\delta_{\mathrm{v}_{1}} L & \cdot \delta v_{1}+\delta_{\mathrm{v}_{2}} L \cdot \delta v_{2} \\
= & \int_{\Gamma_{\text {wall }}} \hat{v}_{1} \delta v_{1}+\hat{v}_{2} \delta v_{2} \mathrm{~d} \Gamma+\int_{\Gamma} \delta v_{1}\left[\frac{\partial j_{\Gamma}}{\partial v_{1}}+\hat{v}_{1} \frac{\partial v_{1}}{\partial x_{1}}+\hat{v}_{1} v_{1} n_{1}\right. \\
& \left.+\hat{v}_{1} v_{2} n_{2}-\hat{p} n_{2}+\nu \frac{\partial \hat{v}_{1}}{\partial x_{2}} n_{2}\right]-\nu\left(\hat{v}_{1} \frac{\partial\left(\delta v_{1}\right)}{\partial x_{2}} n_{2}\right) \\
& +\delta v_{2}\left[\frac{\partial j_{\Gamma}}{\partial v_{2}}-\hat{p} n_{2}\right] \mathrm{d} \Gamma+\int_{\Omega} \delta v_{1}\left[\hat{v}_{1} \frac{\partial v_{1}}{\partial x_{1}}-\frac{\partial \hat{v}_{1} v_{1}}{\partial x_{1}}\right. \\
& \left.-\frac{\partial \hat{v}_{1} v_{2}}{\partial x_{2}}-\nu \frac{\partial^{2} \hat{v}_{1}}{\partial x_{2}^{2}}-\frac{\partial \hat{p}}{\partial x_{1}}+\frac{\partial j_{\Omega}}{\partial v_{1}}\right] \\
& +\delta v_{2}\left[\hat{v}_{1} \frac{\partial v_{1}}{\partial x_{2}}-\frac{\partial \hat{p}}{\partial x_{2}}+\frac{\partial j_{\Omega}}{\partial v_{2}}\right] \mathrm{d} \Omega \\
\stackrel{!}{=} & 0 \forall\left(\delta v_{1}, \delta v_{2}\right), \\
\delta_{\mathrm{p}} L \cdot \delta p & =\int_{\Gamma_{\text {wall }}} \hat{p} \frac{\partial(\delta p)}{\partial n} \mathrm{~d} \Gamma+\int_{\Gamma} \delta p\left[\frac{\partial j_{\Gamma}}{\partial p}+\hat{v}_{1} n_{1} \frac{1}{\rho}+\hat{v}_{2} n_{2} \frac{1}{\rho}\right] \mathrm{d} \Gamma \\
& +\int_{\Omega} \delta p\left[-\frac{\partial \hat{v}_{1}}{\partial x_{1}} \frac{1}{\rho}-\frac{\partial \hat{v}_{2}}{\partial x_{2}} \frac{1}{\rho}\right] \mathrm{d} \Omega \\
& \stackrel{!}{=} 0 \forall \delta p .
\end{aligned}
$$

The adjoint BL equations and their boundary conditions in Table I are obtained from Eqs. (15) and (16). Assuming a sufficiently smooth boundary, a sensitivity rule with respect to a generalized control parameter can be computed based on the remaining first order optimality criteria. Basically, this control-derivative depends on the definition of the control. In the context of shape optimization, we exemplary refer to a shape derivative along the controlled design wall, $24,25,40$

$$
\delta_{u} J=\int_{\Gamma_{\mathrm{D}}} s\left(V_{\mathrm{i}} n_{\mathrm{i}}\right) \mathrm{d} \Gamma_{\mathrm{O}}=\int_{\Gamma_{\mathrm{D}}} s \mathrm{~d} \Gamma_{\mathrm{O}} \quad \text { with } \quad s=-\nu \frac{\partial v_{\mathrm{i}}}{\partial x_{\mathrm{j}}} \frac{\partial \hat{v}_{\mathrm{i}}}{\partial x_{k}} n_{\mathrm{j}} n_{k},
$$

in the direction of a pseudo-velocity field $V_{\mathrm{i}}$ (cf. Ref. 25). In this work, we confine our self to $V_{\mathrm{i}}=n_{\mathrm{i}}$.

\section{DERIVATION OF THE ADJOINT BLASIUS EQUATIONS}

Prior to addressing the adjoint Blasius solution, it is instructive to repeat the fundamentals of the primal Blasius solution in brief. Further details can be found in textbooks, e.g., Ref. 35. We consider the flat plate BL flow where the extent in the streamwise $x_{1}$ direction is much larger than in the $x_{2}$ direction normal to the plate (cf. Fig. 2). The plate length $L$ is expected to tend to infinity, i.e., $L \rightarrow \infty$, and we assume homogeneous steady inflow $V_{1}$. Generalized solutions for Eqs. (3)-(5) try to downgrade the PDEs to ODEs. The latter is achieved based on a coordinate transformation, which in turn needs suited similarity coordinates. For both the primal and the adjoint system, we conceptually split the derivation in two parts: (a) we derive a suitable similarity variable that (b) should simplify the corresponding equations.

\section{A. The primal boundary layer}

Flow is anticipated to be a function of the plate normal and tangential coordinate, viz., $v_{1} / V_{1}=g(\eta)$, with $\eta=x_{2} / \delta\left(x_{1}\right)$, where $g$ and $\delta$ represent a (so far) unknown function and a measure for the BL thickness, respectively. Thus, we can directly compute its spatial derivatives, e.g., $\partial v_{1} / \partial x_{1}=-V_{1} g^{\prime}\left(x_{2} / \delta^{2}\right)\left(\partial \delta / \partial x_{1}\right)$, with $g^{\prime}=\mathrm{d} g / \mathrm{d} \eta$. An integration of Eq. (3) along the wall-normal coordinate from the wall to the BL edge reads

$$
2 \int_{0}^{\delta} v_{1} \frac{\partial v_{1}}{\partial x_{1}} \mathrm{~d} x_{2}+\int_{0}^{\delta} v_{2} \frac{\partial v_{1}}{\partial x_{2}} \mathrm{~d} x_{2}-\int_{0}^{\delta} \nu \frac{\partial^{2} v_{1}}{\partial x_{2}^{2}} \mathrm{~d} x_{2}=0 .
$$

Applying the continuity equation $\left(\partial v_{2} / \partial x_{2}=-\partial v_{1} / \partial x_{1}\right)$ together with the new definition of the plate tangential velocity allows the elimination of the plate-normal velocity, i.e.,

$$
-2 V_{1}^{2} \int_{0}^{\delta} g g^{\prime} \frac{x_{2}}{\delta^{2}} \frac{\partial \delta}{\partial x_{1}} \mathrm{~d} x_{2}+V_{1}^{2} \int_{0}^{\delta} g^{\prime} \frac{x_{2}}{\delta^{2}} \frac{\partial \delta}{\partial x_{1}} \mathrm{~d} x_{2}-\left.\nu V_{1} g^{\prime} \frac{1}{\delta}\right|_{0} ^{\delta}=0 .
$$

Substituting $\eta=x_{2} / \delta\left[\mathrm{d} x_{2}=\delta \mathrm{d} \eta=\mathrm{d}\left(x_{2} / \delta\right)\right]$ offers an ODE for $\delta$, viz.,

$$
[B-2 A] \frac{\partial \delta}{\partial x_{1}} \delta=\frac{\nu}{V_{1}} C,
$$

where all integrals are condensed to the parameters $A$ and $B$; hence,

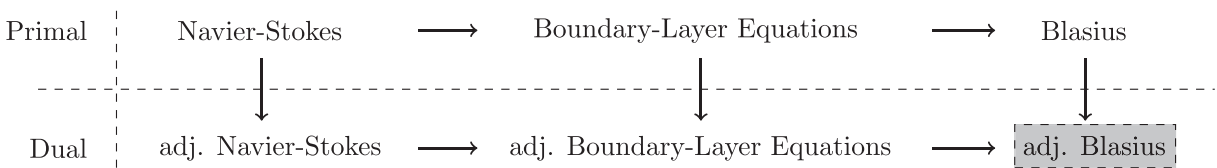

FIG. 1. Schematic derivation flow of adjoint counterparts to known primal simplifications for near-wall flow physics toward the desired adjoint Blasius equation (dark gray). 


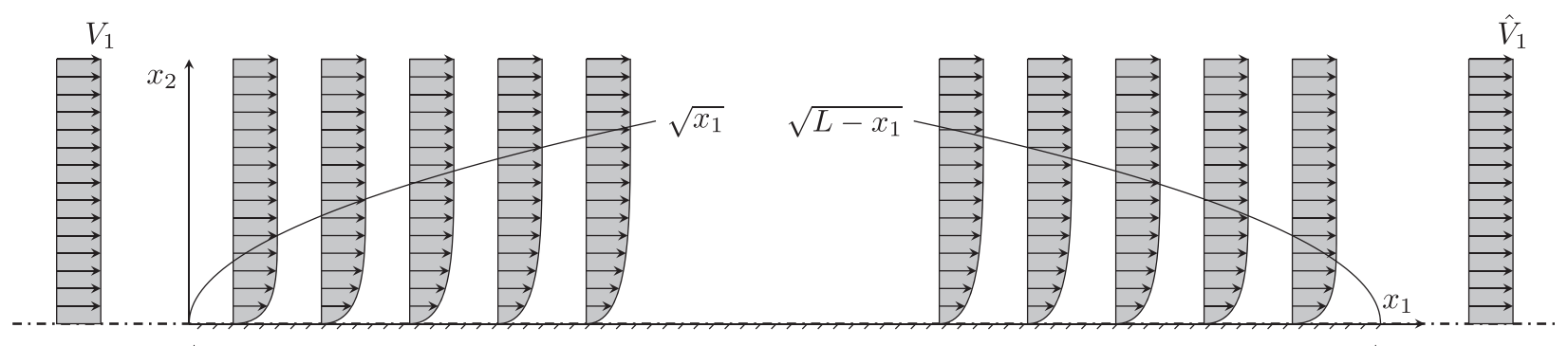

L

FIG. 2. Illustration of the (forward) primal $V_{1}$ and (backward) adjoint $\hat{V}_{1}$ flow over a flat finite plate in the $x_{1}-x_{2}$ plane.

$$
A=\int_{0}^{1} g g^{\prime} \eta \mathrm{d} \eta \quad B=\int_{0}^{1} g^{\prime} \eta \mathrm{d} \eta \quad C=g^{\prime}(1)-g^{\prime}(0) .
$$

Equation (20) can be solved with respect to $\delta$, which yields

$$
\delta=\sqrt{\frac{2 C}{b[B-2 A]}} \sqrt{\frac{\nu\left[a+b x_{1}\right]}{V_{1}}} \Rightarrow \delta \propto \sqrt{\frac{\nu\left[a+b x_{1}\right]}{V_{1}}} .
$$

The last expression supports an estimation of the boundary-layer thickness and offers a suitable choice for the similarity variable $\eta=x_{2} \sqrt{V_{1} /\left[\nu\left(a+b x_{1}\right)\right]}$. It should be pointed out that classical $\mathrm{BL}$ thickness measures employ $a=0$ and $b=1$, which is basically due to the choice for the origin of the coordinate system. However, motivated by the subsequent adjoint analysis, we continue with the more general expression.

Since a suitable similarity variable is found, we seek for a velocity field that satisfies Eqs. (3)-(5). Introducing a stream function $\psi$ that inherently satisfies the continuity expression (5), e.g., $v_{1}=\partial \psi / \partial x_{2}$, $v_{2}=-\partial \psi / \partial x_{1}$, offers access to the plate tangential velocity, viz.,

$$
\psi=\int_{0}^{x_{2}} v_{1} \mathrm{~d} x_{2}=\int_{\eta(0)}^{\eta\left(x_{2}\right)} g(\eta) V_{1} \delta \mathrm{d} \eta=\sqrt{\nu\left[a+b x_{1}\right] V_{1}} \underbrace{\int_{0}^{\eta} g \mathrm{~d} \eta}_{f(\eta)} .
$$

All primal BL terms can be expressed in terms of $f$ and $\eta$, e.g., $\partial v_{1}^{2} / \partial x_{2}^{2}=V_{1}^{2} /\left[\nu\left(a+b x_{1}\right)\right] f^{\prime \prime \prime}$, as summarized in Appendix C. Assuming a homogeneous pressure field, the substitutions of all terms of Eq. (3) yields the well-known Blasius equation

$$
\mathrm{R}_{1}^{\mathrm{v}, \mathrm{BL}} \rightarrow-2 f^{\prime \prime \prime}-b f f^{\prime \prime}=0 .
$$

\section{B. The adjoint boundary layer}

Quantities are convected by the primal flow, and we anticipate a similar behavior of the adjoint field and a scalar field, e.g., a temperature field, although the latter usually does not have to fulfill a continuity equation. The interpretation of the adjoint flow is non-intuitive due to the influence of the objective functional, e.g., volume based objectives possibly introduce a non-divergence free adjoint velocity field. Therefore, we confine the present investigations to boundary based objectives. In line with the primal flow, we start with the similarity variable and assume the adjoint mean flow to depend on the plate normal and tangential coordinate, viz., $\hat{v}_{1} / \hat{V}_{1}=\hat{g}(\hat{\eta})$, with $\hat{\eta}=x_{2} / \hat{\delta}\left(x_{1}\right)$, where $\hat{g}$ and $\hat{\delta}$ represent the adjoint complement of $g$ and $\delta$. Again, we can directly compute all required spatial derivatives, e.g., $\partial \hat{v}_{1} / \partial x_{1}=-\hat{V}_{1} \hat{g}^{\prime}\left(x_{2} / \hat{\delta}^{2}\right)\left(\partial \hat{\delta} / \partial x_{1}\right)$, with $\hat{g}^{\prime}=\mathrm{d} \hat{g} / \mathrm{d} \hat{\eta}$. An integration of (11) between the wall and the adjoint BL edge while assuming adjoint ZPG along the wall normal coordinate reads

$$
\begin{aligned}
& -\int_{0}^{\hat{\delta}} v_{1} \frac{\partial \hat{v}_{1}}{\partial x_{1}} \mathrm{~d} x_{2}-\int_{0}^{\hat{\delta}} v_{2} \frac{\partial \hat{v}_{1}}{\partial x_{2}} \mathrm{~d} x_{2}-\int_{0}^{\hat{\delta}} \nu \frac{\partial^{2} \hat{v}_{1}}{\partial x_{2}^{2}} \mathrm{~d} x_{2} \\
& =-\int_{0}^{\hat{\delta}} \hat{v}_{1} \frac{\partial v_{1}}{\partial x_{1}} \mathrm{~d} x_{2} .
\end{aligned}
$$

Here, the additional term on the right hand side corresponds to the ATC term originating from the non-linear convection. Applying the primal continuity equation $\left(\partial v_{2} / \partial x_{2}=-\partial v_{1} / \partial x_{1}\right)$ interestingly cancels the ATC term, viz.,

$$
\begin{aligned}
& -\int_{0}^{\hat{\delta}} v_{1} \frac{\partial \hat{v}_{1}}{\partial x_{1}} \mathrm{~d} x_{2}+\hat{V}_{1} \int_{0}^{\hat{\delta}} \frac{\partial v_{1}}{\partial x_{1}} \mathrm{~d} x_{2}-\int_{0}^{\hat{\delta}} \hat{v}_{1} \frac{\partial v_{1}}{\partial x_{1}} \mathrm{~d} x_{2}-\left.\nu \frac{\partial \hat{v}_{1}}{\partial x_{2}}\right|_{0} ^{\hat{\delta}} \\
& =-\int_{0}^{\hat{\delta}} \hat{v}_{1} \frac{\partial v_{1}}{\partial x_{1}} \mathrm{~d} x_{2} .
\end{aligned}
$$

Combining the primal tangential velocity $\left[v_{1} / V_{1}=g(\eta)\right]$ and its (anticipated) adjoint complement yields

$$
\frac{\partial \hat{\delta}}{\partial x_{1}} \int_{0}^{1} g \hat{g}^{\prime} \hat{\eta} \mathrm{d} \hat{\eta}-\frac{\partial \delta}{\partial x_{1}} \int_{0}^{\delta / \hat{\delta}} g^{\prime} \eta \mathrm{d} \eta-\left.\frac{\nu}{V_{1}} \hat{g}^{\prime} \frac{1}{\hat{\delta}}\right|_{0} ^{1}=0 .
$$

Substituting $\hat{\eta}=x_{2} / \hat{\delta}\left[\mathrm{d} x_{2}=\hat{\delta} \mathrm{d} \hat{\eta}=\hat{\delta} \mathrm{d}\left(x_{2} / \hat{\delta}\right)\right]$ offers an ODE for $\hat{\delta}$ that inheres the primal BL thickness measure

$$
\left[\hat{A} \frac{\partial \hat{\delta}}{\partial x_{1}}-\hat{B} \frac{\partial \delta}{\partial x_{1}}\right] \hat{\delta}=\frac{\nu}{V_{1}} \hat{C} .
$$

Again, all integrals are condensed into coefficients, viz.,

$$
\hat{A}=\int_{0}^{1} g \hat{g}^{\prime} \hat{\eta} \mathrm{d} \hat{\eta} \quad \hat{B}=\int_{0}^{\delta / \hat{\delta}} g^{\prime} \eta \mathrm{d} \eta \quad \hat{C}=\hat{g}^{\prime}(1)-\hat{g}^{\prime}(0) .
$$

Thanks to the available primal $\delta$, we can reformulate Eq. (28), viz.,

$$
C_{1} \frac{\partial \hat{\delta}}{\partial x_{1}} \hat{\delta}-C_{2} \frac{\hat{\delta}}{\sqrt{a+b x_{1}}}=C_{3}
$$

where $C_{1}=\hat{A}, C_{2}=\hat{B}(b / 2) \sqrt{2 C /(b[A-2 B])} \sqrt{\nu / V_{1}}$, and $C_{3}$ $=\hat{C} \nu / V_{1}$ are introduced to keep the notation compact. Thus, we can solve Eq. (30) with respect to $\hat{\delta}$, 


$$
\hat{\delta}=-\frac{2 C_{3}}{C_{2}+\sqrt{C_{2}^{2}+2 b C_{1} C_{3}}} \sqrt{a+b x_{1}},
$$

and quantify an adjoint BL thickness by the expression

$$
\begin{aligned}
\hat{\delta} & =-\frac{C_{3}}{C_{2}} \frac{b \hat{B}}{C_{2}+\sqrt{C_{2}^{2}+2 b C_{1} C_{3}}} \underbrace{\sqrt{\frac{2 C}{b[B-2 A]}} \sqrt{\frac{\nu(a+b x)}{V_{1}}}}_{\delta} \\
& \Rightarrow \hat{\delta} \propto \delta \propto \sqrt{\frac{\nu(a+b x)}{V_{1}}},
\end{aligned}
$$

which is somehow proportional to the primal BL thickness. Finally, we can define an adjoint similarity variable that equals the primal one, viz.,

$$
\hat{\eta}=\frac{x_{2}}{\hat{\delta}} \propto x_{2} \sqrt{\frac{V_{1}}{\nu\left(a+b x_{1}\right)}}=\frac{x_{2}}{\delta}=\eta .
$$

A challenge in the interpretation of the adjoint results follows from the inverted convection characteristics of the adjoint flow field. To describe the primal BL velocity, the origin of the coordinate system is typically positioned at the plate origin $(a=0, b=1)$. However, a reasonable measurement based on the plate end is obtained for the adjoint system $(\mathrm{a}=\mathrm{L}, \mathrm{b}=-1)$. The latter motivates the negative sign in Eq. (32) and indicates a formal issue for the numerical verification/ validation of the approach: Physically, the plate is assumed to extent infinitely in the streamwise direction. For an adjoint approach, the latter is numerically uncomfortable.

Analogous to the primal derivation, we seek now for an adjoint velocity field that satisfies Eqs. (11)-(13) where the primal flow field is already known. Again, we have to satisfy a continuity equation and therefore define an adjoint stream function $\hat{\psi}$ that inherently complies with (13), e.g., $\hat{v}_{1}=\partial \hat{\psi} / \partial x_{2}, \hat{v}_{2}=-\partial \hat{\psi} / \partial x_{1}$, and offers access to the wall tangential adjoint velocity, viz.,

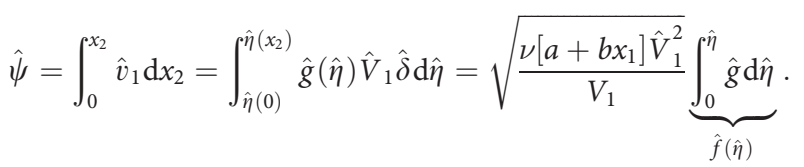

All adjoint $\mathrm{BL}$ expression can be computed from $\hat{f}$ and $\hat{\eta}$, e.g., $\partial \hat{v}_{1}^{2} / \partial x_{2}^{2}=\hat{V}_{1} V_{1} /\left[\nu\left(a+b x_{1}\right)\right] \hat{f}^{\prime \prime \prime}$. Details of the similarity transformations are provided in Appendix C. The streamwise adjoint BL Eq. (11) reduces to

$$
b[\hat{\eta}-\eta] f^{\prime} \hat{f}^{\prime \prime}+b f \hat{f}^{\prime \prime}-2 \hat{f}^{\prime \prime \prime}=b f^{\prime \prime} \hat{f}^{\prime} \eta .
$$

In combination with (33), we achieve the adjoint complement to the Blasius equation

$$
\begin{gathered}
\mathrm{R}_{1}^{\hat{v}, \mathrm{BL}} \rightarrow-2 \hat{f}^{\prime \prime \prime}+b \hat{f}^{\prime \prime} f=b \hat{f}^{\prime} f^{\prime \prime} \eta, \\
\mathrm{R}_{2}^{\hat{v}, \mathrm{BL}} \rightarrow \hat{f}^{\prime} f^{\prime \prime}=0 .
\end{gathered}
$$

The first [second] equation corresponds to the generalized tangential [normal] adjoint $\mathrm{BL}$ equation. Interestingly, the normal adjoint momentum balance (37) cancels the ATC term in the corresponding tangential direction (36). The latter is similar to their primal counterpart with an inverted sign in front of the non-linearity originating from the inverted convection characteristics.

To pursue the simplify-and-derive strategy, one typically first inserts the primal simplification. As a result, however, the streamwise adjoint Blasius equation (36) cannot be retrieved directly from a variation of (24) due to the ( $x_{1}$-nonlinear) $\eta$-based coordinate transformation inherent to the initial simplification step. A way out avoids the similarity transformation and declares the $x_{2}$-derivative of the adjoint stream function $\hat{\psi}_{x_{2}}=\partial \hat{\psi} / \partial x_{2}$ as the Lagrange multiplier. The latter represents an educated guess, which follows from the previous discussions of the paper, viz.,

$$
\begin{gathered}
L=\ldots+\int_{\mathrm{x}_{1}} \int_{\mathrm{x}_{2}} \hat{v}_{1} \mathrm{R}_{1}^{\mathrm{v}, \mathrm{BL}} \mathrm{d} x_{2} \mathrm{~d} x_{1} \\
\rightarrow \quad L=\ldots+\int_{\mathrm{x}_{1}} \int_{\mathrm{x}_{2}} \hat{\psi}_{x_{2}}\left[\psi_{x_{2}} \psi_{x_{2}, x_{1}}-\psi_{x_{1}} \psi_{x_{2}, x_{2}}\right. \\
\left.\quad+\nu \psi_{x_{2}, x_{2}, x_{2}}\right] \mathrm{d} x_{2} \mathrm{~d} x_{1} .
\end{gathered}
$$

Using first order optimality conditions, the adjoint equations can be derived from the stream function based formulation (38) using integration by parts in Euclidean (Cartesian) space,

$$
\begin{aligned}
& \delta_{\psi_{\left(x_{2}\right)}} L \cdot \delta\left(\psi_{x_{2}}\right)=\ldots+\int_{\mathrm{x}_{1}} \int_{\mathrm{x}_{2}} \delta\left(\psi_{x_{2}}\right)\left[\hat{\psi}_{x_{2}} \psi_{x_{2}, x_{1}}\right. \\
& -\left(\hat{\psi}_{x_{2}} \psi_{x_{2}}\right)_{x_{1}}-\left(\hat{\psi}_{x_{2}} \psi_{x_{1}}\right)_{x_{2}} \\
& \left.+\nu \hat{\psi}_{x_{2}, x_{2}, x_{2}}\right] \mathrm{d} x_{2} \mathrm{~d} x_{1}, \\
& \delta_{\psi_{\left(x_{1}\right)}} L \cdot \delta\left(\psi_{x_{1}}\right)=\ldots-\int_{\mathrm{x}_{1}} \int_{\mathrm{x}_{2}} \delta\left(\psi_{x_{1}}\right)\left[\hat{\psi}_{x_{2}} \psi_{x_{2}, x_{2}}\right] \mathrm{d} x_{2} \mathrm{~d} x_{1} .
\end{aligned}
$$

We subsequently impose a similarity transformation based on available primal and adjoint similarity relations, viz.,

$$
\begin{gathered}
\delta_{f^{\prime}} L \cdot \delta\left(f^{\prime}\right)=\ldots+\int_{\mathrm{x}_{1}} \int_{\eta} \delta\left(f^{\prime}\right)\left[-b \hat{f}^{\prime} f^{\prime \prime} \eta+b \hat{f}^{\prime \prime} f-2 \hat{f}^{\prime \prime \prime}\right] \\
\quad \times \mathrm{d} \eta \sqrt{\frac{V_{1}^{3} \hat{V}_{1}^{2} \nu}{a+b x_{1}}} \mathrm{~d} x_{1} \stackrel{!}{=} 0 \quad \forall \delta\left(f^{\prime}\right), \\
\delta_{f^{\prime} \eta-f} L \cdot \delta\left(f^{\prime} \eta-f\right)= \\
\times+\int_{\mathrm{x}_{1}} \int_{\eta} \delta\left(f^{\prime} \eta-f\right)\left[\hat{f}^{\prime} f^{\prime \prime}\right] \\
\times \mathrm{d} \eta \sqrt{\frac{V_{1}^{3} \hat{V}_{1}^{2} \nu}{a+b x_{1}}} \mathrm{~d} x_{1} \stackrel{!}{=} 0 \quad \forall \delta\left(f^{\prime} \eta-f\right) .
\end{gathered}
$$

The expressions in the square brackets correspond to the plate tangential and normal adjoint Blasius equations (36) and (37). The first terms in the respective brackets contain the first derivative $\hat{f}^{\prime}$ and originate from the variation of the convection $\left[\left(\delta v_{\mathrm{k}}\right) \partial v_{1} / \partial x_{\mathrm{k}}\right]$. These terms result in the ATC contribution to the adjoint Blasius equations. The term that inheres the second derivative $\hat{f}^{\prime \prime}$ follows from the perturbed convected primal momentum $\left[v_{\mathrm{k}} \partial\left(\delta v_{1}\right) / \partial x_{\mathrm{k}}\right]$ and switches sign due to integration by parts. The diffusion term refers to the third term in (41) and enters the equation analogous to the primal counterpart with a third derivative. Its self-adjoint character is underlined by its consistent sign in the adjoint and primal equations. 


\section{NUMERICAL SOLUTION OF THE ADJOINT BLASIUS EQUATIONS}

The primal/adjoint Blasius equations are numerically approximated based on a shooting method. An exemplary Matlab code is available in Ref. 23. The primal procedure tries to hit the boundary value $f_{\infty}^{\prime}=1$ for $\eta \rightarrow \infty$ with prescribed wall values $f_{0}=0$ and $f_{0}^{\prime}=0$. The boundary value problem is controlled by $f_{0}^{\prime \prime}$ at the wall and iterated to convergence until the value $f_{\infty}^{\prime}$ falls below a numerical limit of $\left(f_{\infty}^{\prime}-1\right)<10^{-08}$. The result is depicted in the left graph of Fig. 3, where $a=0$ and $b=1$ were assumed. The displayed numerical results perfectly match with available data from the literature. ${ }^{5,35}$

The adjoint solution employs stored discrete values of the primal procedure. It uses the same discretization of the generalized similarity variable $\eta$ together with a similar shooting approach to compute the adjoint Blasius solution. Using $a=L$ and $b=-1$, the method aims at hitting the value $\hat{f}_{\infty}^{\prime}=1$ for $\eta \rightarrow \infty$ with prescribed wall values $\hat{f}_{0}=0$ and $\hat{f}_{0}^{\prime}=0$ but variable $\hat{f}_{0}^{\prime \prime}$ for $\eta=0$. Similar to the primal flow, the adjoint boundary value problem is iterated to convergence until $\left(\hat{f}^{\prime}-1\right)<10^{-8}$ is reached. The numerical results are shown in the right graph of Fig. 3 for two simulations that either consider or neglect the ATC term. In the case of neglecting ATC, the procedure resembles the primal results. As we will show in Sec. V, the latter is expected from analytical studies. In the case with ATC, the convergence of $\hat{f}_{\infty}^{\prime}$ is shifted outwards by one order of magnitude in $\eta$ and results in an increased BL thickness.

\section{ANALYTICAL INVESTIGATION OF THE ADJOINT BLASIUS EQUATIONS}

Using the similarity transformation introduced in Sec. III, the adjoint BL Eqs. (11)-(13) were successfully transformed from PDEs into ODEs. Only the surface-based objective functional is considered in this paper, for which Eqs. (36) and (37) are generally valid. The

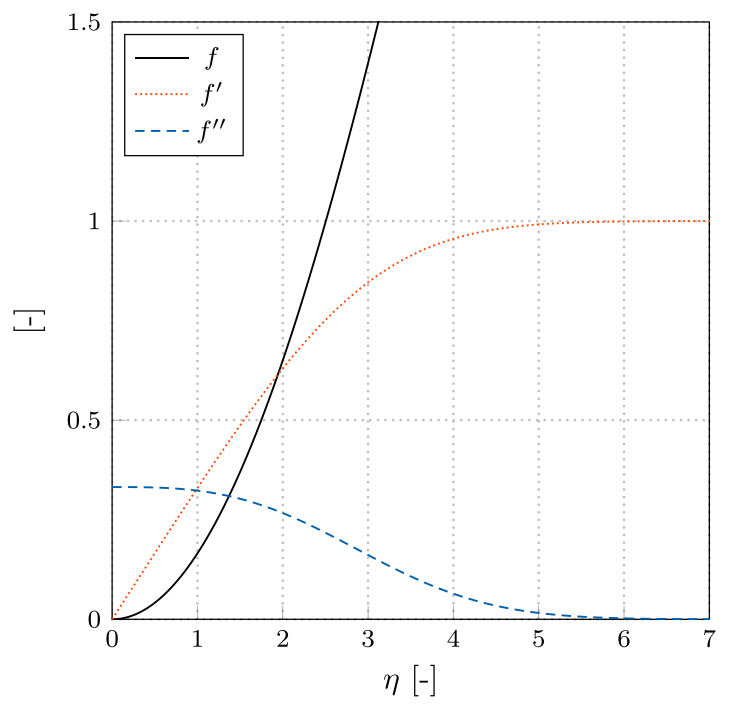

(a) derivation of the continuous adjoint equations reveals the use of Dirichlet conditions for the adjoint velocity along no-slip walls (cf. Table I). The latter follows from the objective of interest, which, however, can occur in different forms depending on the underlying objective functional.

In the following, we assume either a linear dependence of the functional with respect to pressure or no functional along the plate. An illustrative example refers to the evaluation of flow induced forces on the considered plate, where two options to introduce this objective are conceivable. Using an internal formulation, the stresses $\left[p \delta_{i k}-2 \nu S_{\mathrm{ik}}\right] d_{\mathrm{i}}$ are first projected in a prescribed spatial direction $d_{\mathrm{i}}$ and subsequently integrated over (a part of) the plate $\left(\Delta \Gamma_{k}\right)$. On the contrary, an external formulation balances the momentum loss between the inlet and the outlet. From a physical perspective, both approaches pose the same question. However, the adjoint answer is fundamentally different since the habitat of the objective is either the internal wall along the plate or the external inlet/outlet area. Hence, we end up with different boundary conditions for the wall value, i.e., $\hat{v}_{\mathrm{i}}=-d_{\mathrm{i}}$ or $\hat{v}_{\mathrm{i}}=0$ for the interior and the exterior approach, respectively. The different approaches resemble the transport theorem formulated in an Arbitrary Lagrangian Eulerian (ALE) frame of reference as indicated in Fig. 7, and we refer to Kühl et al. ${ }^{25}$ for a more detailed discussion. Despite these differences, both formulations employ the similarity transformation to derive a generalized velocity profile, and we can define a normalized adjoint tangential velocity profile using

$$
\hat{f}^{\prime}=\frac{\hat{v}_{1}}{\hat{V}_{1}} \rightarrow \hat{f}^{\prime}=\frac{\hat{v}_{1}-\hat{V}_{1, \mathrm{w}}}{\hat{V}_{1, \infty}-\hat{V}_{1, \mathrm{w}}} .
$$

Equation (43) reminds of the Blasius solution for a thermal BL, e.g., $-2 \Theta^{\prime \prime}-\operatorname{Pr} f \Theta^{\prime}=0$, where $\Theta=\left(T-T_{\mathrm{w}}\right) /\left(T_{\infty}-T_{\mathrm{w}}\right)$ is a nondimensional temperature and $T$ and Pr represent the temperature and Prandtl number.

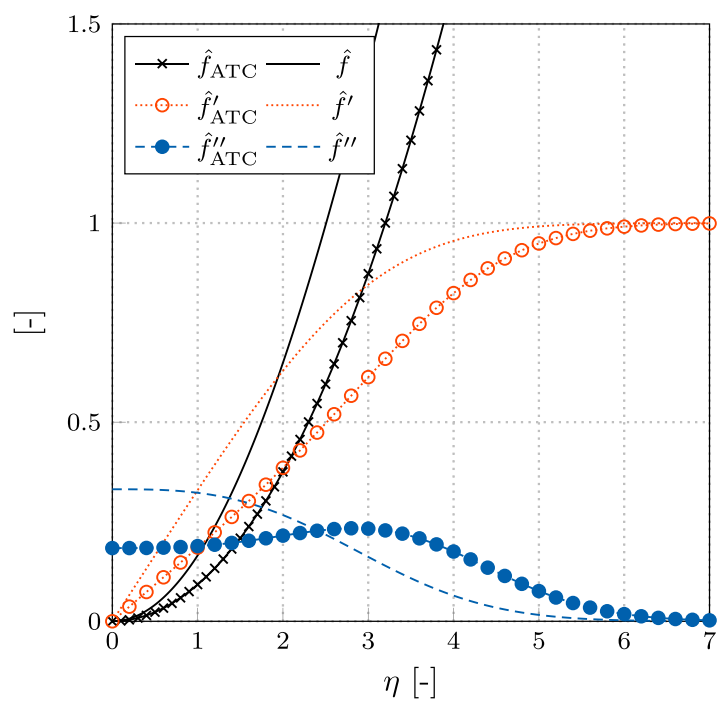

(b)

FIG. 3. Results of a shooting method for (a) the primal and (b) the adjoint Blasius equation. Marked lines in (b) indicate adjoint results considering the adjoint transpose convection (ATC) term. 


\section{A. Influence of adjoint transposed convection}

Inserting the normal adjoint Blasius equation (37) into its tangential companion yields the adjoint Blasius Eq. (36) without ATC on the right hand side. Choosing a BL measure based on $a=0$ and $b=1$, we can incorporate the primal Blasius expression $\left(f=-2 f^{\prime \prime \prime} / f^{\prime \prime}\right)$ into the adjoint counterpart and conclude

$$
\frac{f^{\prime \prime \prime}}{f^{\prime \prime}}=-\frac{\hat{f}^{\prime \prime \prime}}{\hat{f}^{\prime \prime}} .
$$

We explicitly point out that expression (44) agrees with an analogue relationship of the thermal BL, viz.,

$$
\frac{f^{\prime \prime \prime}}{f^{\prime \prime}}=\frac{1}{\operatorname{Pr}} \frac{\Theta^{\prime \prime}}{\Theta^{\prime}}
$$

for a negative unity Prandtl number $\operatorname{Pr}=-1$, which underlines the reverse flow direction of adjoint systems. Separation of variables allows for a solution for $\hat{f}^{\prime}$ and $\Theta$,

$$
\hat{f}^{\prime}=C_{1} \int_{0}^{\eta} \frac{1}{f^{\prime \prime}} \mathrm{d} \eta+C_{2} \quad \text { and } \quad \Theta=C_{3} \int_{0}^{\eta}\left(f^{\prime \prime}\right)^{\operatorname{Pr}} \mathrm{d} \eta+C_{4} .
$$

Unfortunately, the negative unit Prandtl number of the adjoint system introduces a singularity in (46) since $f^{\prime \prime} \rightarrow 0$ for $\eta \rightarrow \infty$ holds for the primal system as shown in Sec. IV. The singularity can only be circumvented with an appropriate measure for the adjoint $\mathrm{BL}$, viz., $a=L$ and $b=-1$. The integration constants of the adjoint velocity profile follow from the boundary conditions for $\hat{f}^{\prime}(\eta \rightarrow 0)=0$ and $\hat{f}^{\prime}(\eta \rightarrow \infty)$ $=1$ and yield

$$
C_{1}=\int_{0}^{\infty} f^{\prime \prime} \mathrm{d} \eta \quad \text { and } \quad C_{2}=0
$$

The final solution reads

$$
\hat{f}^{\prime}=\frac{\int_{0}^{\eta} f^{\prime \prime} \mathrm{d} \eta}{\int_{0}^{\infty} f^{\prime \prime} \mathrm{d} \eta} \rightarrow \hat{f}^{\prime}=\frac{f^{\prime}}{f^{\prime}(\infty)-f^{\prime}(0)}=f^{\prime} .
$$

Hence, the generalized adjoint velocity profile equals its primal counterpart when ATC is suppressed.

\section{Estimation of (dual) interface thickness and wall shear stress}

For known values of the Blasius solution, various statements about, e.g., BL thickness or shear stress distribution can be derived. This also applies to the adjoint counterparts. Assigning the primal BL thickness to $v_{1} / V_{1}=0.99$ yields a value of $\eta_{99} \rightarrow 4.91 \approx 5$ in line with Fig. 3 and Eq. (33),

$$
\delta_{99} \approx 5 \sqrt{\frac{\nu\left(a+b x_{1}\right)}{V_{1}}} \text { or } \frac{\delta_{99}}{\left(a+b x_{1}\right)} \approx \frac{5}{\sqrt{\operatorname{Re}_{\left(\mathrm{a}+\mathrm{bx}_{1}\right)}}} .
$$

The numerical and analytical results of Secs. IV and V A reveal the same BL thickness for primal and adjoint flows, viz., $\hat{\delta}_{99}=\delta_{99}$ in the case of no ATC. However, if the additional right hand side term is incorporated, we end up with approximately $\hat{\eta}_{99} \rightarrow 5.9424 \approx 6$, cf. Fig. 3, and thus,

$$
\hat{\delta}_{99} \approx 6 \sqrt{\frac{\nu\left(a+b x_{1}\right)}{V_{1}}} \text { or } \frac{\hat{\delta}_{99}}{\left(a+b x_{1}\right)} \approx \frac{6}{\sqrt{\operatorname{Re}_{\left(\mathrm{a}+\mathrm{bx}_{1}\right)}}} .
$$

In the remainder of this subsection, the estimated adjoint Blasius values refer to the formulation including ATC, and the index $(\cdot)_{\mathrm{w}}$ indicates an evaluation along the wall at $\eta=0$. Note that neglecting the ATC yields strong similarities between the primal and dual Blasius solutions, e.g., $\hat{f}_{\mathrm{w}}^{\prime \prime}=f_{\mathrm{w}}^{\prime \prime}$ (cf. Sec. V). Several primal BL thickness measures exist, e.g., the displacement $\left(\delta_{\mathrm{D}}\right)$, momentum $\left(\delta_{\mathrm{M}}\right)$, and energy $\left(\delta_{\mathrm{E}}\right)$ thickness, which follow from the relation $\mathrm{d} x_{2}=\delta \mathrm{d} \eta$

$$
\begin{aligned}
& V_{1} \delta_{\mathrm{D}}=\int_{0}^{\infty}\left[V_{1}-v_{1}\right] \mathrm{d} x_{2} \rightarrow \delta_{\mathrm{D}}=\int_{0}^{\infty}\left[1-f^{\prime}\right] \mathrm{d} x_{2} \\
& \rightarrow \quad \delta_{\mathrm{D}} \approx 1.7208 \sqrt{\frac{\nu\left(a+b x_{1}\right)}{V_{1}}}, \\
& \rho V_{1}^{2} \delta_{\mathrm{M}}=\rho \int_{0}^{\infty} v_{1}\left[V_{1}-v_{1}\right] \mathrm{d} x_{2} \rightarrow \delta_{\mathrm{M}}=\int_{0}^{\infty} f^{\prime}\left[1-f^{\prime}\right] \mathrm{d} x_{2} \\
& \rightarrow \delta_{\mathrm{M}} \approx 0.6641 \sqrt{\frac{\nu\left(a+b x_{1}\right)}{V_{1}}}, \\
& \rho V_{1}^{3} \delta_{\mathrm{E}}=\rho \int_{0}^{\infty} v_{1}\left[V_{1}^{2}-v_{1}^{2}\right] \mathrm{d} x_{2} \rightarrow \delta_{\mathrm{E}}=\int_{0}^{\infty} f^{\prime}\left[1-f^{\prime 2}\right] \mathrm{d} x_{2} \\
& \rightarrow \delta_{\mathrm{E}} \approx 1.0444 \sqrt{\frac{\nu\left(a+b x_{1}\right)}{V_{1}}} .
\end{aligned}
$$

Similar expressions can be derived for the adjoint BL, viz.,

$$
\begin{aligned}
\hat{V}_{1} \hat{\delta}_{\mathrm{D}} & =\int_{0}^{\infty}\left[\hat{V}_{1}-\hat{v}_{1}\right] \mathrm{d} x_{2} \rightarrow \hat{\delta}_{\mathrm{D}}=\int_{0}^{1}\left[1-\hat{f}^{\prime}\right] \mathrm{d} x_{2} \\
& \rightarrow \hat{\delta}_{\mathrm{D}} \approx 2.5336 \sqrt{\frac{\nu\left(a+b x_{1}\right)}{V_{1}}}, \\
\rho \hat{V}_{1}^{2} \hat{\delta}_{\mathrm{M}} & =\rho \int_{0}^{\infty} \hat{v}_{1}\left[\hat{V}_{1}-\hat{v}_{1}\right] \mathrm{d} x_{2} \rightarrow \hat{\delta}_{\mathrm{M}}=\int_{0}^{1} \hat{f}^{\prime}\left[1-\hat{f}^{\prime}\right] \mathrm{d} x_{2} \\
& \rightarrow \hat{\delta}_{\mathrm{M}} \approx 0.8430 \sqrt{\frac{\nu\left(a+b x_{1}\right)}{V_{1}}}, \\
\rho \hat{V}_{1}^{3} \hat{\delta}_{\mathrm{E}} & =\rho \int_{0}^{\infty} \hat{v}_{1}\left[\hat{V}_{1}^{2}-\hat{v}_{1}^{2}\right] \mathrm{d} x_{2} \rightarrow \hat{\delta}_{\mathrm{E}}=\int_{0}^{1} \hat{f}^{\prime}\left[1-\hat{f}^{\prime, 2}\right] \mathrm{d} x_{2} \\
& \rightarrow \hat{\delta}_{\mathrm{E}} \approx 1.2830 \sqrt{\frac{\nu\left(a+b x_{1}\right)}{V_{1}}} .
\end{aligned}
$$

The relations between primal and adjoint BL thicknesses read

$$
\begin{aligned}
& \frac{\delta_{99}}{\hat{\delta}_{99}} \approx 0.8263, \quad \frac{\delta_{\mathrm{D}}}{\hat{\delta}_{\mathrm{D}}} \approx 0.6792, \quad \frac{\delta_{\mathrm{M}}}{\hat{\delta}_{\mathrm{M}}} \approx 0.7878, \\
& \text { and } \frac{\delta_{\mathrm{E}}}{\hat{\delta}_{\mathrm{E}}} \approx 0.8140 .
\end{aligned}
$$

Additionally, the generalized Blasius solution offers insights into the primal and adjoint shear stresses acting on the plate, 


$$
\begin{gathered}
\tau_{\mathrm{w}}=\left.\mu \frac{\partial v_{1}}{\partial x_{2}}\right|_{\mathrm{x}_{2}=0}=\mu V_{1} \sqrt{\frac{V_{1}}{\nu\left(a+b x_{1}\right)} f_{\mathrm{w}}^{\prime \prime}} \\
\rightarrow \quad \tau_{\mathrm{w}} \approx 0.3321 \mu V_{1} \sqrt{\frac{V_{1}}{\nu\left(a+b x_{1}\right)}}, \\
\hat{\tau}_{\mathrm{w}}=\left.\mu \frac{\partial \hat{v}_{1}}{\partial x_{2}}\right|_{\mathrm{x}_{2}=0}=\mu \hat{V}_{1} \sqrt{\frac{V_{1}}{\nu\left(a+b x_{1}\right)} \hat{f}_{\mathrm{w}}^{\prime \prime}} \\
\rightarrow \hat{\tau}_{\mathrm{w}} \approx 0.1845 \mu \hat{V}_{1} \sqrt{\frac{V_{1}}{\nu\left(a+b x_{1}\right)}} .
\end{gathered}
$$

The resulting dual shear is significantly smaller compared to the primal one, e.g., $\tau_{\mathrm{w}} / \hat{\tau}_{\mathrm{w}}=f_{\mathrm{w}}^{\prime \prime} / \hat{f}_{\mathrm{w}}^{\prime \prime}\left(V_{1} / \hat{V}_{1}\right)$. The shear is usually nondimensionalized via a primal $\left(\rho V_{1}^{2} / 2\right)$ or an adjoint $\left(\rho V_{1} \hat{V}_{1} / 2\right)$ dynamic pressure to obtain skin-friction coefficients, i.e.,

$$
\begin{gathered}
c_{\mathrm{f}}=\frac{\tau_{\mathrm{w}}}{\frac{1}{2} \rho V_{1}^{2}}=\frac{2 f_{\mathrm{w}}^{\prime \prime}}{\sqrt{\mathrm{Re}_{\mathrm{a}+\mathrm{bx}_{1}}}} \rightarrow c_{\mathrm{f}} \approx \frac{0.6642}{\sqrt{\mathrm{Re}_{\mathrm{a}+\mathrm{bx}_{1}}}}, \\
\hat{c}_{\mathrm{f}}=\frac{\hat{\tau}_{\mathrm{w}}}{\frac{1}{2} \rho \hat{V}_{1} V_{1}}=\frac{2 \hat{f}_{\mathrm{w}}^{\prime \prime}}{\sqrt{\mathrm{Re}_{\mathrm{a}+\mathrm{bx}_{1}}}} \rightarrow \hat{c}_{\mathrm{f}} \approx \frac{0.369}{\sqrt{\mathrm{Re}_{\mathrm{a}+\mathrm{bx}_{1}}}} .
\end{gathered}
$$

Moreover, known shear-stress distributions allow the integration of a total shear forces on the plate,

$$
\begin{aligned}
F_{\mathrm{s}} & =\int_{\Gamma} \tau_{\mathrm{w}} \mathrm{d} \Gamma_{\mathrm{w}}=\left.t \int_{0}^{\mathrm{L}} \mu \frac{\partial v_{1}}{\partial x_{2}}\right|_{\mathrm{x}_{2}=0} \mathrm{~d} x_{1} \\
& =2 f_{\mathrm{w}}^{\prime \prime} t V_{1} \sqrt{\mu \rho V_{1}} \frac{\sqrt{a+b L}-\sqrt{a}}{b} \\
& \rightarrow \quad F_{\mathrm{s}} \approx 0.664 t V_{1} \sqrt{\mu \rho V_{1}} \frac{\sqrt{a+b L}-\sqrt{a}}{b}, \\
\hat{F}_{\mathrm{s}} & =\int_{\Gamma} \hat{\tau}_{\mathrm{w}} \mathrm{d} \Gamma_{\mathrm{w}}=\left.t \int_{0}^{\mathrm{L}} \mu \frac{\partial \hat{v}_{1}}{\partial x_{2}}\right|_{\mathrm{x}_{2}=0} \mathrm{~d} x_{1} \\
& =2 \hat{f}_{\mathrm{w}}^{\prime \prime} t \hat{V}_{1} \sqrt{\mu \rho V_{1}} \frac{\sqrt{a+b L}-\sqrt{a}}{b} \\
& \rightarrow \quad \hat{F}_{\mathrm{s}} \approx 0.369 t \hat{V}_{1} \sqrt{\mu \rho V_{1}} \frac{\sqrt{a+b L}-\sqrt{a}}{b},
\end{aligned}
$$

where $t$ corresponds to the lateral expansion of the plate. As expected, the choice of the coordinate system has no influence on the forces since $F_{\mathrm{s}}(a=0, b=1)=F_{\mathrm{s}}(a=L, b=-1)=2 f_{\mathrm{w}}^{\prime \prime} t \sqrt{\mu \rho L V_{1}^{3}}$ and $\hat{F}_{s}(a=0, b=1)=\hat{F}_{\mathrm{s}}(a=L, b=-1)=2 \hat{f}_{\mathrm{w}}^{\prime \prime} t \sqrt{\mu \rho L V_{1} \hat{V}_{1}^{2}}$. Hence, the ratio between primal and dual shear reads $F_{\mathrm{s}} / \hat{F}_{\mathrm{s}}=f_{\mathrm{w}}^{\prime \prime} / \hat{f}_{\mathrm{w}}^{\prime \prime}$ $\left(V_{1} / \hat{V}_{1}\right)$. We can compute the primal [adjoint] drag coefficient either from an integration of Eqs. (60) and (61) or from dividing Eqs. (62) and (63) by the dynamic pressure times wetted surface $L t$, viz.,

$$
\begin{aligned}
c_{\mathrm{d}} & =\frac{F_{\mathrm{s}}}{p_{\infty} t L}=\frac{1}{L} \int_{0}^{\mathrm{L}} c_{\mathrm{f}} \mathrm{d} x_{1}=\frac{4 f_{\mathrm{w}}^{\prime \prime}}{\sqrt{R e_{\mathrm{L}}}} \frac{\sqrt{a+b L}-\sqrt{a}}{b \sqrt{L}} \\
\rightarrow \quad c_{\mathrm{d}} & \approx \frac{1.3284}{\sqrt{R e_{\mathrm{L}}}} \frac{\sqrt{a+b L}-\sqrt{a}}{b \sqrt{L}},
\end{aligned}
$$

$$
\begin{aligned}
\hat{c}_{\mathrm{d}} & =\frac{\hat{F}_{\mathrm{s}}}{\hat{p}_{\infty} t L}=\frac{1}{L} \int_{0}^{\mathrm{L}} \hat{c}_{\mathrm{f}} \mathrm{d} x_{1}=\frac{4 \hat{f}_{\mathrm{w}}^{\prime \prime}}{\sqrt{R e_{\mathrm{L}}}} \frac{\sqrt{a+b L}-\sqrt{a}}{b \sqrt{L}} \\
\rightarrow \quad \hat{c}_{\mathrm{d}} & \approx \frac{0.738}{\sqrt{R e_{\mathrm{L}}}} \frac{\sqrt{a+b L}-\sqrt{a}}{b \sqrt{L}} .
\end{aligned}
$$

Except different scaling, the dual quantities resemble the wellknown primal relationship.

According to Eq. (17), the combination of primal and dual shear results in a sensitivity distribution along the design surface of the shape. Analogous to the simplification of the shear objective $\left(J=F_{\mathrm{s}}\right)$, the approach also applies to its shape sensitivity, viz.,

$$
\begin{aligned}
s= & -\nu \frac{\tau_{\mathrm{w}}}{\mu} \frac{\hat{\tau}_{\mathrm{w}}}{\mu}=-\left.\left.\nu \frac{\partial v_{1}}{\partial x_{2}}\right|_{\mathrm{x}_{2}=0} \frac{\partial \hat{v}_{1}}{\partial x_{2}}\right|_{\mathrm{x}_{2}=0}=-f_{\mathrm{w}}^{\prime \prime} \hat{f}_{\mathrm{w}}^{\prime \prime} \frac{\hat{V}_{1} V_{1}^{2}}{a+b x_{1}} \\
& \rightarrow s \approx-0.06127 \frac{\hat{V}_{1} V_{1}^{2}}{a+b x_{1}} .
\end{aligned}
$$

The local shape derivative has again singularities at the leading and trailing edges of the plate. However, the intermediate behavior scales with $x^{-1}$ instead of $x^{-1 / 2}$. Furthermore, the local sensitivity increases quadratically with the primal but only linearly with the adjoint velocity. This reminds of the quadratic character of the primal NS equations (e.g., $v_{\mathrm{k}} \partial v_{\mathrm{i}} / \partial x_{\mathrm{k}}$ ), which are opposed by the linear nature of the adjoint counterpart (e.g., $v_{\mathrm{k}} \partial \hat{v}_{\mathrm{i}} / \partial x_{\mathrm{k}}$ ). The sensitivity expression (66) can be non-dimensionalized toward a sensitivity-coefficient $c_{s}$ via the kinematic viscosity and the primal and dual dynamic pressures or by combining the primal and adjoint skin-friction coefficients, viz.,

$$
\begin{aligned}
c_{\mathrm{s}} & =s \frac{\rho^{2} \nu}{p_{\infty} \hat{p}_{\infty}}=s \frac{4 \nu}{V_{1}^{3} \hat{V}_{1}}=-c_{\mathrm{f}} \hat{c}_{\mathrm{f}}=-\frac{4 f_{\mathrm{w}}^{\prime \prime} \hat{f}_{\mathrm{w}}^{\prime \prime}}{\mathrm{Re}_{\mathrm{a}+\mathrm{bx}_{1}}} \\
& \rightarrow \quad c_{\mathrm{s}} \approx-\frac{0.06127}{\mathrm{Re}_{\mathrm{a}+\mathrm{bx}_{1}}} .
\end{aligned}
$$

Finally, the integration of (66) along the plate provides the sensitivity derivative at an integral level,

$$
\begin{aligned}
\delta_{\mathrm{u}} J & =t \int_{0}^{L} s \mathrm{~d} x_{1}=-f_{\mathrm{w}}^{\prime \prime} \hat{f}_{\mathrm{w}}^{\prime \prime} \frac{t \hat{V}_{1} V_{1}^{2}}{b} \ln \left[\frac{a+b L}{a}\right] \\
& \rightarrow \quad \delta_{\mathrm{u}} J \approx-0.06127 \frac{t \hat{V}_{1} V_{1}^{2}}{b} \ln \left[\frac{a+b L}{a}\right] .
\end{aligned}
$$

Interestingly, a singularity arises for the integral sensitivity. Owing to the proportionality $s \propto x^{-1}$, a logarithmic $\delta_{\mathrm{u}} J \propto \pm(\ln (L)-\ln (0))$ relationship results in the integral sensitivity. The $s \propto x^{-1}$ relationship originates in the definition of the similarity variable, which in turn estimates the primal interface thickness by $\delta \propto x^{1 / 2}$ that finally yields $s \propto 1 / \delta^{2}$. The singularity cannot be avoided by adjusting the coordinate system (i.e., $a$ and $b$ ) since the integral bounds also need to be adjusted to $a$ and $b$. Mind that we apply only $a=0, b=1$ or $a=L, b=-1$ in this manuscript. It seems that the plate has an infinite potential to reduce its flow resistance from an integral point of view. This statement seems suspicious at first. However, mind that a disappearing plate would wipe out its resistance completely. A perturbation into the plate normal affects the drag via a variation in the local shear $\delta F \propto \delta f_{\mathrm{w}}^{\prime \prime}$ [cf. Eq. (62)], which in turn follows from a variation in the similarity variable $\delta f_{\mathrm{w}}^{\prime \prime} \rightarrow f_{\mathrm{w}}^{\prime \prime \prime} \delta \eta$ that can finally be estimated via $\delta \eta \propto \delta x_{2} / \sqrt{x_{1}}-0.5\left(\eta / x_{1}\right) \delta x_{1}$ for an interface measure that employs 
$a=0$ and $b=1$. Thus, $\delta F \propto f_{\mathrm{w}}^{\prime \prime \prime}\left[\delta x_{2} / \sqrt{x_{1}}-0.5\left(\eta / x_{1}\right) \delta x_{1}\right]$ becomes singular if both coordinates tend to zero. Note that the drag and the similarity variable become singular at the leading edge and the influence of a plate-normal variation is debatable, not least because the direction of the normal is not defined at the leading edge.

The integral shape derivative can be non-dimensionalized based on the dynamic pressures and the wetted surface. An alternative approach to a global sensitivity coefficient follows from the integration over the sensitivity coefficient, viz.,

$$
\begin{aligned}
c_{\delta_{\mathrm{u}} \mathrm{J}} & =\frac{\delta_{\mathrm{u}} J \nu}{p_{\infty} \hat{p}_{\infty} t L}=-\frac{1}{L} \int_{0}^{\mathrm{L}} c_{\mathrm{s}} \mathrm{d} x_{1}=-\frac{f_{\mathrm{w}}^{\prime \prime} \hat{f}_{\mathrm{w}}^{\prime \prime}}{\operatorname{Re}_{\mathrm{L}}} \frac{1}{b} \ln \left[\frac{a+b L}{a}\right] \\
& \rightarrow \quad c_{\delta_{\mathrm{u}} \mathrm{J}} \approx-\frac{0.06127}{\operatorname{Re}_{\mathrm{L}}} \frac{1}{b} \ln \left[\frac{a+b L}{a}\right] .
\end{aligned}
$$

However, we would like to point out that an interpretation of adjoint values, regarding, e.g., units or the definition of a sensitivity rule, depends strongly on the objective under investigation. The same holds for the definition of the control.

\section{APPLICATIONS}

This section aims at the numerical assessment of the theoretical observations reported in the previous chapters. For this reason, the flow over a simple, finite-length flat plate is considered, cf. Figs. 2 and 4. The computational studies are performed for laminar flows at Reynolds numbers between $10^{3} \leq \operatorname{Re}_{\mathrm{L}}=V_{1} L / \nu \leq 10^{5}$ based on a homogeneous flow velocity $V_{1}$ and the kinematic viscosity $\nu$ using a Navier-Stokes procedure.

\section{A. Numerical procedure}

The numerical procedure is based upon the Finite-Volume procedure FreSCo+. ${ }^{34}$ Analogous to the use of integration-by-parts in deriving the continuous adjoint equations, summation-by-parts is employed to derive the building blocks of the discrete (dual) adjoint expressions. A detailed derivation of this hybrid adjoint approach can be found in Refs. 21, 24, and 40. The segregated algorithm uses a cellcentered, collocated storage arrangement for all transport properties. The implicit numerical approximation is second order accurate and supports polyhedral cells. The primal and adjoint pressure-velocity coupling is based on a SIMPLE method, and possible parallelization is realized by means of a domain decomposition approach. ${ }^{44,45}$ In terms of a CAD-free shape optimization approach, the computational grid can be adjusted using a Laplace-Beltrami ${ }^{22,40}$ or Steklov-Poincaré en $^{2437}$ type (surface metric) approach.

\section{B. Computational model and numerical grid}

The two-dimensional domain has a length and a height of $3 \mathrm{~L}$ and $L$. The inlet and top boundaries are located one length away from the origin of the coordinate system (cf. Fig. 4). The latter is located in the leading edge of the plate. The velocity is prescribed at the inlet, a slip wall is used along the top boundary, and symmetry conditions are employed along the bottom before and after the plate. Zero gradient conditions are employed at the outlet. The convective term for primal [adjoint] momentum is approximated using the Quadratic Upstream [Downstream] Interpolation of Convective Kinematics (QUICK) [QU(D)ICK] scheme.

To ensure the independence of the objective functional with respect to spatial discretization, a grid study was conducted. The considered five grids are all symmetric with respect to the mid-plate at $x_{1}=L / 2$, and the grid spacing in horizontal and vertical direction was successively halved between two consecutive grids. Figure 5 depicts the evolution of the drag coefficient $c_{\mathrm{d}}$ over the grid refinement level-indicated by the number of control volumes $n_{\mathrm{fv}}$-for an exemplary flow at $\mathrm{Re}_{\mathrm{L}}=10^{4}$. Based on the grid convergence studies, all results shown hereafter were obtained for the finest grid level that consists of approximately 28.000 control volumes. The controlled plate
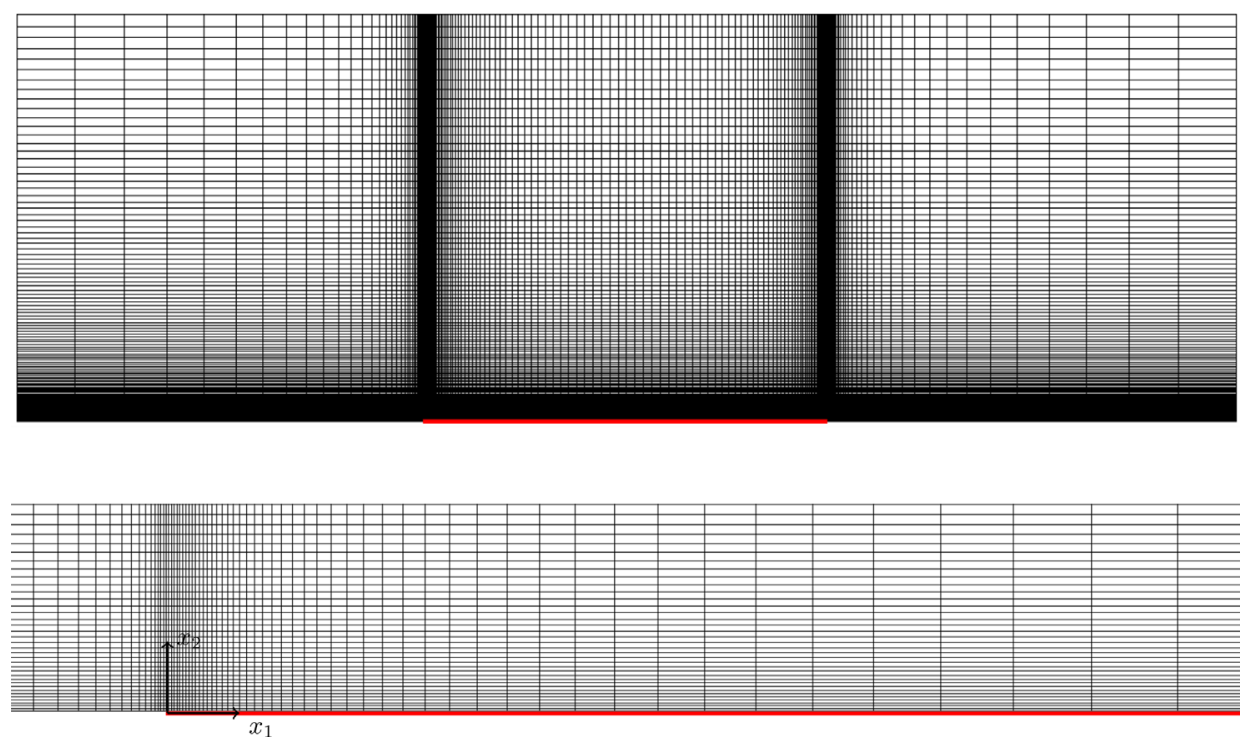

FIG. 4. Employed structured grid for the flat plate flow. Complete domain (top) and refined region around the leading edge where the origin of the coordinate system is located (bottom). The plate is indicated by red lines. 

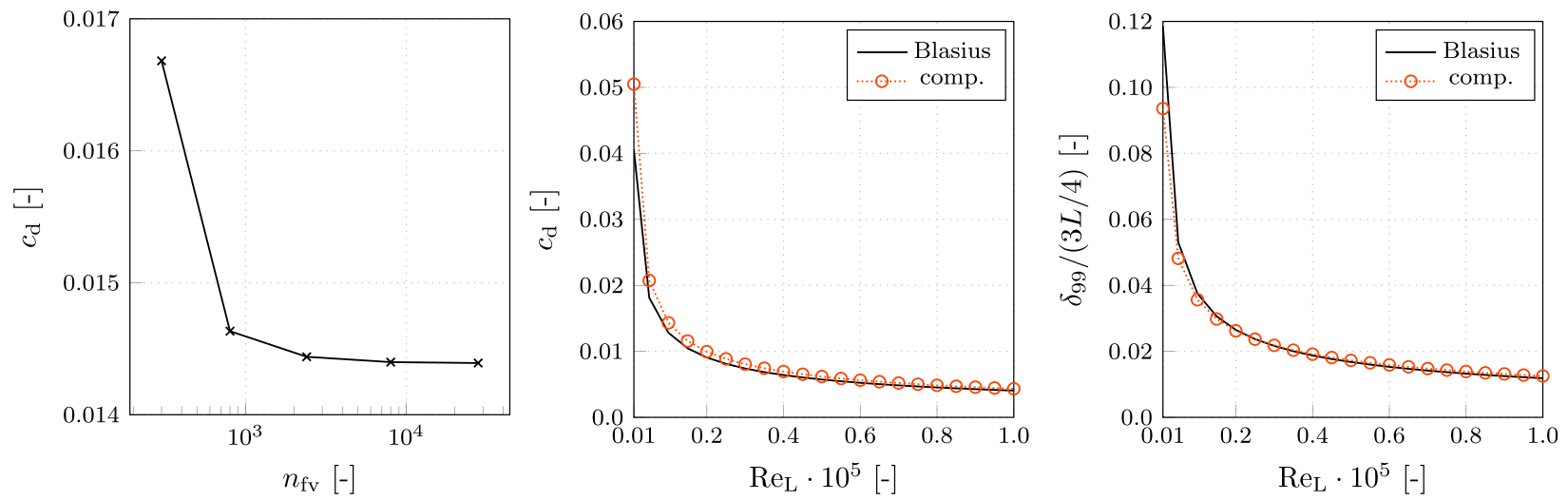

FIG. 5. Predicted integral parameters for the primal flat plate flow: evolution of the frag coefficient $c_{d}$ over the grid refinement level-indicated by the amount of control volumes $-n_{\mathrm{fv}}$ (left), comparison of Blasius and numerical drag coefficient, as well as BL thickness $\left(\delta_{99}\right)$ predictions at $x / L=3 / 4$ for a range of Reynolds numbers (center, right).

shape is discretized with 160 surface elements, and the boundary layers were typically resolved by more than 50 control volumes. Since the investigated Reynolds numbers vary by two orders of magnitude, the plate normal resolution was adjusted to ensure $y^{+}=\mathcal{O}\left(10^{-1}\right)$ in all cases.

\section{Primal flow results}

The quality of adjoint results hinges on the quality of the preceding primal approximation. Therefore, first the predictive accuracy of the estimates made in Sec. VAl is compared against primal Navier-Stokes results. Figure 5 compares the predicted results for drag coefficient $c_{\mathrm{d}}$ (center) and 99\%-BL thickness $\delta_{99}$ at $x_{1} / L=3 / 4$ (right) against the Blasius results based on a measure that employs $a=0$ and $b=1$. Quantitatively, the resistance coefficient [interface thickness] obtained from a Blasius solution is slightly overestimated [underestimated] for small Reynolds numbers, whereby the qualitative behavior is still in fair agreement.

Supplementary to the comparison of integral parameters depicted in Fig. 5, local results were examined. The results displayed in the present paper are confined to $\mathrm{Re}_{\mathrm{L}}=10^{4}$. Figure 6 compares the normalized tangential $v_{1} / V_{1}$ (left) and normal $v_{2} / V_{1}$ (center) velocity profiles against the similarity solution for a range of locations. While the tangential velocity fits almost perfectly with the Blasius solution, the normal component deviates significantly from the expected solution when the $\mathrm{BL}$ approaches the trailing edge. This phenomenon is attributed to the abrupt change of the boundary condition for the examined finite-length plate (cf. Fig. 4) and the ability of trailing-edge information to propagate upstream in a Navier-Stokes framework. As also shown in Fig. 6, pronounced deviations from Blasius solutions occur for the local skin-friction coefficient $c_{\mathrm{f}}$ at the trailing edge and approximately $20 \%$ upstream.

\section{Adjoint flow results}

The adjoint investigations were performed for a drag objective $\left(d_{\mathrm{i}}=[1,0]^{\mathrm{T}}\right)$ on the basis of two different formulations referred to as interior (FI) or exterior (FE) drag-force evaluation (cf. Sec. V). Additionally, we distinguish between simulations that neglect $(\mathrm{A} 0)$ or employ (A1) ATC. Thus, four adjoint computations were conducted for each primal flow.

\section{Unified adjoint velocity profile}

As shown in Sec. V, the internal and external adjoint formulation of a force functional can be unified. To illustrate this, the results obtained from an exemplary adjoint simulation at $\operatorname{Re}_{\mathrm{L}}=10^{4}$ are discussed in more detail. Figure 7 illustrates predicted adjoint tangential velocity profiles at ten equally spaced positions along the plate for both the FI and FE formulation. Both sets of exemplary results incorporate ATC (A1). As outlined in Sec. V, the FI and FE adjoint velocity profiles reveal a similarity since they are based on different formulations that aim at answering the same engineering question. Optically visible and numerically measurable, both adjoint velocity profile sets grow out of the plate with the same gradient. The latter enters the sensitivity along the plate $\left(x_{2} / L=0\right)$ according to Eq. (17). In addition to the adjoint velocity profiles, the $99 \%$-BL thickness $\hat{\delta}_{99}$ is depicted for various formulations, viz., [FI, FE] $\times[\mathrm{A} 0, \mathrm{~A} 1]$. The trend of an increased $\hat{\delta}_{99}$ BL thickness due to the influence of ATC (A0 vs A1) is reproduced by the simulations, though to a lesser extent.

\section{Manipulated primal field}

The investigated boundary layer contradicts the assumption of an infinitely long plate immanent to the theory, and deviations between the computed Navier-Stokes results and the Blasius solution increase toward the trailing edge of the plate. However, due to the (approximately) parabolic nature of the computed flow, deviations are primarily transported downstream, and their significant local influence documented in Fig. 6 has hardly any effect at an integral level (cf. Fig. 5). A different picture emerges for the adjoint flow. Since the adjoint solution propagates from the trailing to the leading edge, the processes reverse their direction in adjoint mode, and primal flow deviations are introduced at the adjoint upstream location. Therefore, the comparison of computed and Blasius adjoint results for a plate of finite length is afflicted by initial value deviations of the primal field and might be debatable. For this reason, the primal velocity field at $\operatorname{Re}_{\mathrm{L}}=10^{4}$ was manipulated by reinitializing a velocity field that follows from the similarity transformation of a computed primal velocity 

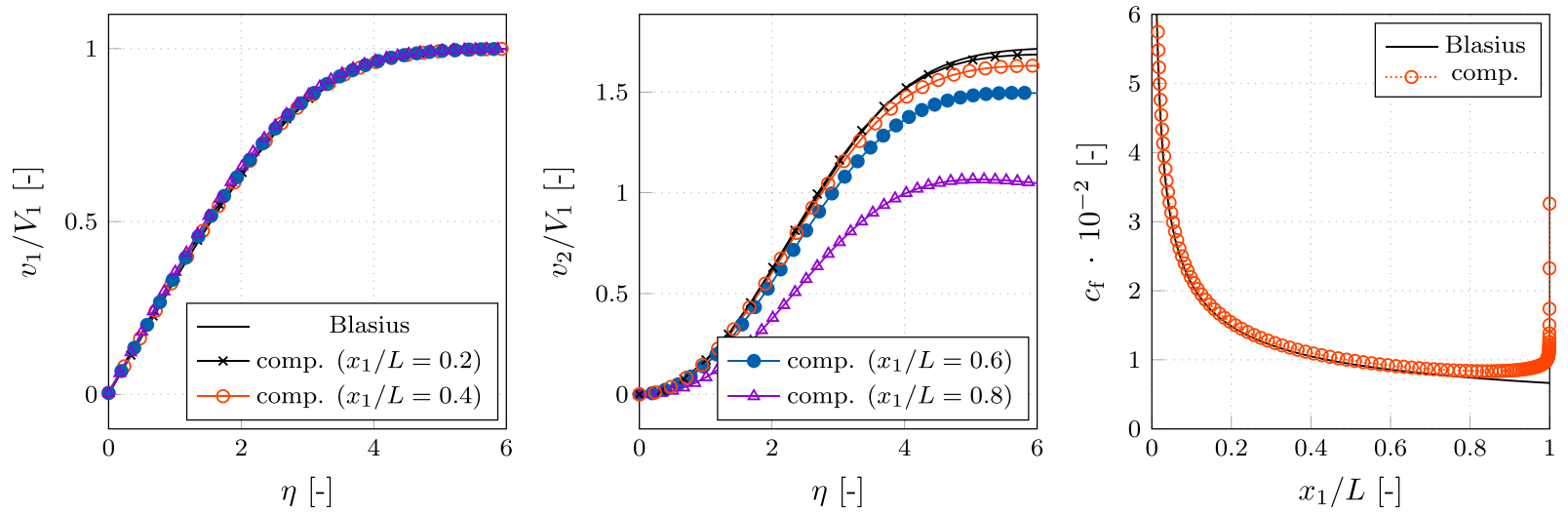

FIG. 6. Comparison of Blasius and Navier-Stokes results for the primal flow over a flat plate at $\operatorname{Re}_{\mathrm{L}}=10^{4}$ : tangential velocity $\left(v_{1} / V_{1}\right)$ against $f^{\prime}$ (left) and normal velocity $\left(v_{2} / V_{1}\right)$ against $f^{\prime} \eta-f$ (center) at four different locations and skin-friction coefficient $c_{\mathrm{f}}$ against $0.664 / \operatorname{Re}_{x_{1}}$ based on $a=0$ and $b=1$ (right).

profile extracted at $\mathrm{x} / \mathrm{L}=3 / 4$. Subsequently, all four adjoint simulations were performed based on $a=L$ and $b=-1$. Figure 8 outlines the computed results for the normalized adjoint tangential $\hat{v}_{1} / \hat{V}_{1}$ (left) and normal $\hat{v}_{2} / \hat{V}_{1}$ (center) velocity next to the adjoint friction coefficient (right) in comparison to the Blasius solutions with (A1) and without (A0) ATC. Only minor deviations are observed when comparing the Navier-Stokes predictions for different adjoint formulations. However, an improved agreement with the Blasius solution that neglects the ATC (A0) is clearly seen. This supports the results of the similarity transformation, i.e., Eqs. (36) and (37), according to which ATC influence should disappear in laminar flat plate BL flows. Analogous to the primal investigation, the plate tangential adjoint velocity profiles are closer to their companion Blasius solutions than the normal velocity profiles. However, a smaller normal velocity variation is observed between the different locations. Moreover, the manipulation of the (upstream) primal results reveals benefits for the agreement between the Blasius and the computed adjoint skin friction.

\section{Consistent primal field}

The final subsection discusses computed adjoint Navier-Stokes results based upon non-manipulated predictions of the primal flow, which are displayed in Figs. 5 and 6. Figure 9 shows the evolution of the adjoint drag-coefficient $\hat{c}_{\mathrm{d}}$ (left) and the adjoint BL thickness $\hat{\delta}_{99}$ (center) over the Reynolds number for the two considered adjoint formulations (A0 and A1). The adjoint BL thickness is evaluated at $x_{1} / L=1 / 4$ based on a measure that employs $a=L$ and $b=-1$. Regarding the adjoint drag coefficient, the predicted results of the formulation without ATC (A0) are again significantly closer to the corresponding Blasius results than those that include ATC (A1). As already shown in Fig. 7 for $\operatorname{Re}_{\mathrm{L}}=10^{4}$, simulations without adjoint advection result in a reduced $\mathrm{BL}$ thickness in line with the observations of Sec. IV. The investigations in Sec. V reveal that the integral shape sensitivity scales with $1 / \operatorname{Re}_{\mathrm{L}}$ but approaches a singularity toward the leading edge of the plate. Therefore, no adjoint Blasius solutions are shown in Fig. 9 (right). Instead, the numerical results expressed by the dimensionless sensitivity coefficient are compared with two reference curves, which scale with $1 / \operatorname{Re}_{\mathrm{L}}$ and $1 / \sqrt{\operatorname{Re}_{\mathrm{L}}}$, respectively. The computed Navier-Stokes results fall in between these two reference curves but tend to follow the inverse root relation. Due to the more singular behavior in comparison to, e.g., the adjoint drag coefficient $\left[\mathcal{O}\left(-\mathrm{Re}_{\mathrm{L}}^{-1}\right)\right.$ vs $\left.\mathcal{O}\left(-\mathrm{Re}_{\mathrm{L}}^{-1 / 2}\right)\right]$, only the range between $10^{3} \leq \mathrm{Re}_{\mathrm{L}}$ $\leq 5 \times 10^{4}$ is shown. From these results, we conclude that consistent, real (finite-length) plate investigations with respect to integral adjoint quantities are qualitatively in good agreement with analytical relations from Sec. V. Quantitative discrepancies follow mainly from initial offsets due to pronounced local inaccuracies of primal results introduced in the vicinity of the (finite) end of the plate (cf. right graph of Fig. 6).

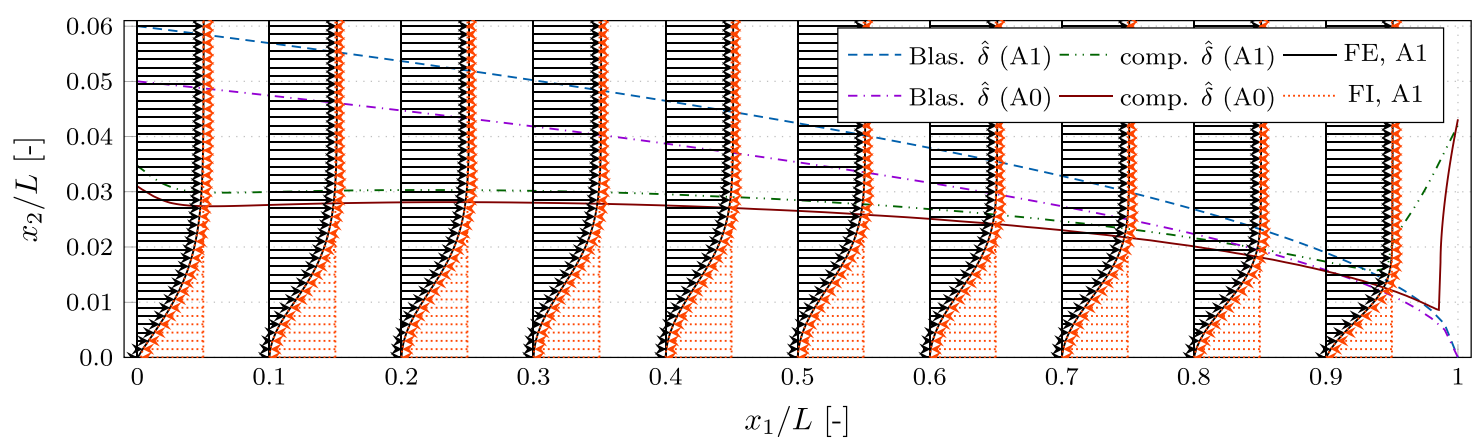

FIG. 7. Comparison of several plate-tangential adjoint velocity profiles $\hat{v}_{1}\left(x_{2}\right)$ for external $\left[\mathrm{FE}, \hat{v}_{1}(0) / \hat{V}_{1}=0\right]$ and internal $\left[\mathrm{FI}, \hat{v}_{1}(0) / \hat{V}_{1}=-1\right]$ drag formulation. Additionally, several adjoint $99 \%$-boundary-layer thicknesses $\hat{\delta}_{99}$ are depicted, augmented by the expected adjoint Blasius solutions. 

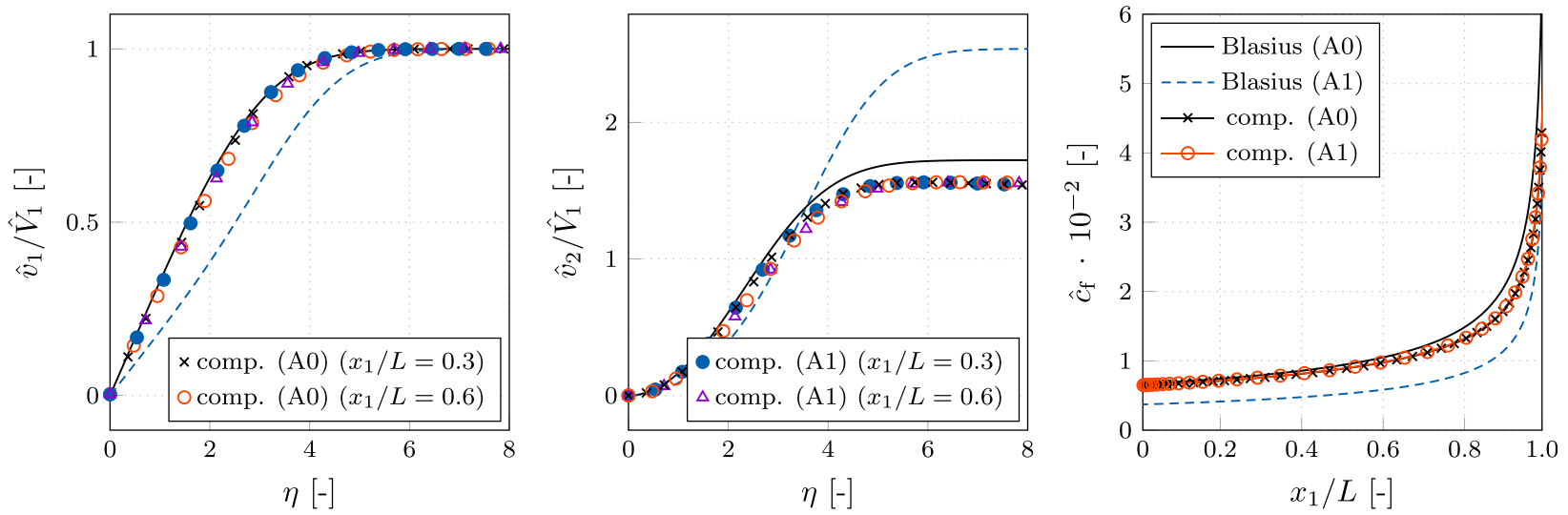

FIG. 8. Local results for the adjoint flow over a flat plate at $\operatorname{Re}_{\mathrm{L}}=10^{4}$ based on a manipulated primal field: (left) tangential adjoint velocity $\left(\hat{v}_{1} / \hat{V}_{1}\right)$ against $\hat{f}^{\prime}$ and $($ center) normal adjoint velocity $\left(\hat{v}_{2} / \hat{V}_{1}\right)$ against $\hat{f} \hat{\eta}-\hat{f}$ both over the similarity variable. (Right) Adjoint friction coefficient $\hat{c}_{f}$ against the adjoint Blasius estimations based on an interface measure that employs $a=L$ and $b=-1$.

\section{CONCLUSIONS AND OUTLOOK}

This paper reports the derivation and analysis of a continuous adjoint complement to the Blasius equation for a flat plate boundary layer. The discussion is divided into three main sections and arrives at two major conclusions.

The first part demonstrated two alternatives for deriving the adjoint boundary-layer equations, either following a simplify-and-derive or a derive-and-simplify strategy. The procedure reminds of two prominent classical adjoint strategies, frequently subdivided into discrete adjoint (discretize-and-derive) vs continuous adjoint (derive-and-discretize).

The second part was devoted to the analysis of the coupled primal/ adjoint BL framework and led to generalized similarity parameters. The first major conclusion refers to the existence of virtually identical similarity parameters to condense the primal and adjoint BL flows. The latter turn the partial-differential-equation problem into a boundary value problem described by a set of ordinary-differential-equations and support the formulation of an adjoint complement to the classical Blasius equation. Opposite to the primal Blasius equation, its adjoint complement consists of two ODEs, which can be simplified depending on the treatment of adjoint advection also known as adjoint transpose convection. It was shown that the advective fluxes vanish for the investigated self-similar BL flows. The second major conclusion supports the heuristic neglect of the term used by many authors of continuous adjoint optimization studies into complex engineering shear flows. Moreover, a formalism was derived for analytical expressions of an adjoint BL thickness, wall shear stress, skin friction, and drag coefficient, as well as a shape sensitivity expression for a shear driven drag objective.

The third part assessed the predictive agreement between the different Blasius solutions and numerical results for Navier-Stokes simulations of a flat plate $\mathrm{BL}$ at Reynolds numbers between $10^{3} \leq \operatorname{Re}_{\mathrm{L}} \leq 10^{5}$. The reversal of the inlet and outlet locations, as well as the direction of the flow, inherent to the adjoint formulation of convective kinematics, poses a challenge when investigating real finite length flat plate boundary layer flows. The neglect of the adjoint transposed convection term was confirmed by the NS results, and the predicted adjoint integral quantities agree with adjoint Blasius results.

Future work should investigate other self-similar flows, e.g., derive adjoint Falkner-Skan solutions for wedged geometries, and
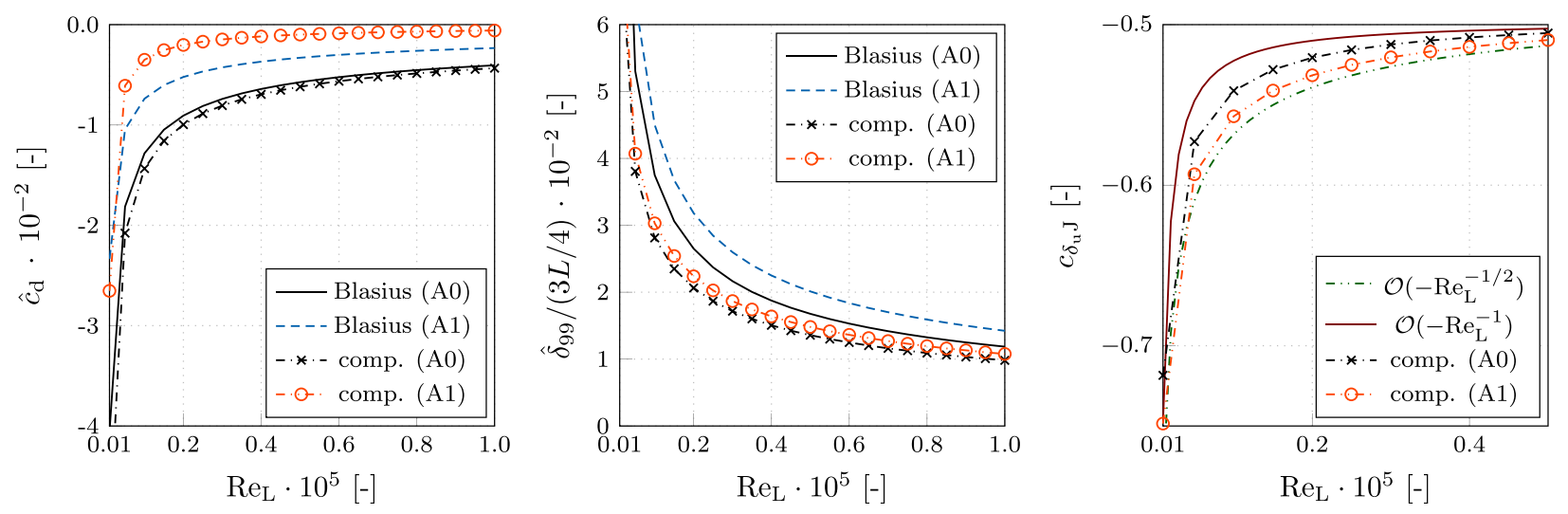

FIG. 9. Integral results for the adjoint flow over a flat plate: (left) adjoint drag coefficient $\hat{c}_{d}$, (center) adjoint boundary-layer thickness $\left(\hat{\delta}_{99}\right)$ at $x / L=1 / 4$, and (right) integral shape sensitivity coefficient $c_{\delta_{\Perp}}$ over the plate-length based Reynolds-number $\mathrm{Re}_{\llcorner}$. 
develop an adjoint solution for compressible or even three dimensional boundary layers. Volume-based objective functionals could be investigated, e.g., to account for variations in the primal flow field, which potentially build a bridge toward the stability crisis immanent to flow transition. Finally, research toward turbulent BL flows might be fruitful, whereby one could follow both the continuous and discrete adjoint approaches in parallel.

\section{ACKNOWLEDGMENTS}

This work is a part of the research projects "Drag Optimisation of Ship Shapes" funded by the German Research Foundation (DFG, Grant No. RU 1575/3-1) and "Dynamic Adaptation of Modular Shape Optimization Processes" funded by the German Federal Ministry for Economic Affairs and Energy (BMWi, Grant No. 03SX453B). Moreover, the research takes places within the Research Training Group (RTG) 2583 "Modeling, Simulation and Optimization with Fluid Dynamic Applications" funded by the German Research Foundation. This support is gratefully acknowledged by the authors.

\section{AUTHORS' CONTRIBUTIONS}

N.K. conceptualized the study, provided methodology and software, performed validation, formal analysis, investigation, and visualization, wrote the original draft, and wrote, reviewed, and edited the manuscript; P.M.M. used methodology, performed formal analysis, and wrote, reviewed, and edited the manuscript; and T.R. administered the project, acquired funding, supervised, provided resources, and wrote, reviewed, and edited the manuscript.

\section{APPENDIX A: PRIMAL SCALING ANALYSIS}

Performing a non-dimensionalization of Eqs. (1) and (2) with the reference data given in Table II results in

$$
\begin{gathered}
\mathrm{R}_{1}^{\mathrm{v} *}: v_{1}^{*} \frac{\partial v_{1}^{*}}{\partial x_{1}^{*}} \frac{V_{1} V_{1}}{L}+v_{2}^{*} \frac{\partial v_{1}^{*}}{\partial x_{2}^{*}} \frac{V_{2} V_{1}}{\delta}+\frac{P}{L \rho} \frac{\partial p^{*}}{\partial x_{1}^{*}} \\
-\nu \frac{V_{1}}{L^{2}}\left[\frac{\partial^{2} v_{1}^{*}}{\partial x_{1}^{* 2}}-\frac{L^{2}}{\delta^{2}} \frac{\partial^{2} v_{1}^{*}}{\partial x_{2}^{* 2}}\right]=0, \\
\mathrm{R}_{2}^{\mathrm{V} *}: v_{1}^{*} \frac{\partial v_{2}^{*}}{\partial x_{1}^{*}} \frac{V_{1}}{L}+v_{2}^{*} \frac{\partial v_{2}^{*}}{\partial x_{2}^{*}} \frac{V_{2}}{\delta}+\frac{P}{\delta \rho} \frac{\partial p^{*}}{\partial x_{2}^{*}} \\
-\nu \frac{V_{2}}{L^{2}}\left[\frac{\partial^{2} v_{2}^{*}}{\partial x_{1}^{* 2}}+\frac{L^{2}}{\delta^{2}} \frac{\partial^{2} v_{2}^{*}}{\partial x_{2}^{* 2}}\right]=0, \\
\mathrm{R}^{\mathrm{p} *}:-\frac{\partial v_{1}^{*}}{\partial x_{1}^{*}} \frac{V_{1}}{L}-\frac{\partial v_{2}^{*}}{\partial x_{2}^{*}} \frac{V_{2}}{\delta}=0 .
\end{gathered}
$$

which can be simplified toward

$$
\begin{gathered}
\mathrm{R}_{1}^{\mathrm{v} *}: v_{1}^{*} \frac{\partial v_{1}^{*}}{\partial x_{1}^{*}}+v_{2}^{*} \frac{\partial v_{1}^{*}}{\partial x_{2}^{*}}+\mathrm{Eu} \frac{\partial p^{*}}{\partial x_{1}^{*}}-\frac{1}{\mathrm{Re}_{\mathrm{L}}}\left[\frac{\partial^{2} v_{1}^{*}}{\partial x_{1}^{* 2}}+\frac{\partial^{2} v_{1}^{*}}{\partial x_{2}^{* 2}} \frac{L^{2}}{\delta^{2}}\right]=0, \quad(\mathrm{~A} 4) \\
\mathrm{R}_{2}^{\mathrm{v} *}: v_{1}^{*} \frac{\partial v_{2}^{*}}{\partial x_{1}^{*}}+v_{2}^{*} \frac{\partial v_{2}^{*}}{\partial x_{2}^{*}}+\mathrm{Eu} \frac{L^{2}}{\delta^{2}} \frac{\partial p^{*}}{\partial x_{2}^{*}}-\frac{1}{\operatorname{Re}_{\mathrm{L}}}\left[\frac{\partial^{2} v_{2}^{*}}{\partial x_{1}^{* 2}}+\frac{\partial^{2} v_{2}^{*}}{\partial x_{2}^{* 2}} \frac{L^{2}}{\delta^{2}}\right]=0,
\end{gathered}
$$

$$
\mathrm{R}^{\mathrm{p} *}:-\frac{\partial v_{1}^{*}}{\partial x_{1}^{*}}-\frac{\partial v_{2}^{*}}{\partial x_{2}^{*}}=0,
$$

by assuming $V_{2} \propto V_{1} \delta / L$ and defining the Reynolds- and Euler numbers as $\operatorname{Re}_{\mathrm{L}}=\frac{L V_{1}}{\nu}$ and $\mathrm{Eu}=\frac{P}{\rho V_{1}^{2}}$, respectively.

\section{APPENDIX B: ADJOINT SCALING ANALYSIS}

Performing a non-dimensionalization of Eqs. (9) and (10) with the reference data given in Table II results in

$$
\begin{aligned}
& \mathrm{R}_{1}^{\hat{v} *}:-v_{1}^{*} \frac{\partial \hat{v}_{1}^{*}}{\partial x_{1}^{*}} \frac{V_{1} \hat{V}_{1}}{L}-v_{2}^{*} \frac{\partial \hat{v}_{1}^{*}}{\partial x_{2}^{*}} \frac{V_{2} \hat{V}_{1}}{\hat{\delta}}+\hat{v}_{1}^{*} \frac{\partial v_{1}^{*}}{\partial x_{1}^{*}} \frac{\hat{V}_{1} V_{1}}{L} \\
& +\hat{v}_{2}^{*} \frac{\partial v_{2}^{*}}{\partial x_{1}^{*}} \frac{\hat{V}_{2} V_{2}}{L}+\frac{\hat{P}}{L} \frac{\partial \hat{p}^{*}}{\partial x_{1}^{*}}-\nu \frac{\hat{V}_{1}}{L^{2}}\left[\frac{\partial^{2} \hat{v}_{1}^{*}}{\partial x_{1}^{* 2}}+\frac{L}{\hat{\delta}} \frac{\partial^{2} \hat{v}_{1}^{*}}{\partial x_{2}^{* 2}}\right] \\
& =-\frac{\partial j_{\Omega}^{*}}{\partial v_{1}^{*}} \frac{J_{\Omega}}{V_{1}} \\
& \mathrm{R}_{2}^{\hat{v} *}:-v_{1}^{*} \frac{\partial \hat{v}_{2}^{*}}{\partial x_{1}^{*}} \frac{V_{1} \hat{V}_{2}}{L}-v_{2}^{*} \frac{\partial \hat{v}_{2}^{*}}{\partial x_{2}^{*}} \frac{V_{2} \hat{V}_{2}}{\hat{\delta}}+\hat{v}_{1}^{*} \frac{\partial v_{1}^{*}}{\partial x_{2}^{*}} \frac{\hat{V}_{1} V_{1}}{\delta} \\
& +\hat{v}_{2}^{*} \frac{\partial v_{2}^{*}}{\partial x_{2}^{*}} \frac{\hat{V}_{2} V_{2}}{\delta}+\frac{\hat{P}}{\hat{\delta}} \frac{\partial \hat{p}^{*}}{\partial x_{2}^{*}}-\nu \frac{\hat{V}_{2}}{L^{2}}\left[\frac{\partial^{2} \hat{v}_{2}^{*}}{\partial x_{1}^{* 2}}+\frac{L}{\hat{\delta}} \frac{\partial^{2} \hat{v}_{2}^{*}}{\partial x_{2}^{* 2}}\right] \\
& =-\frac{\partial j_{\Omega}^{*}}{\partial v_{2}^{*}} \frac{J_{\Omega}}{V_{2}}, \\
& \mathrm{R}^{\hat{p}^{*}}:-\frac{1}{\rho} \frac{\partial \hat{v}_{1}^{*}}{\partial x_{1}^{*}} \frac{\hat{V}_{1}}{L}-\frac{1}{\rho} \frac{\partial \hat{v}_{2}^{*}}{\partial x_{2}^{*}} \frac{\hat{V}_{2}}{\hat{\delta}}=0,
\end{aligned}
$$

which can be simplified toward

$$
\begin{aligned}
& \mathrm{R}_{2}^{\hat{v} *}:-v_{1}^{*} \frac{\partial \hat{v}_{1}^{*}}{\partial x_{1}^{*}}-v_{2}^{*} \frac{\partial \hat{v}_{1}^{*}}{\partial x_{2}^{*}} \frac{\delta}{\hat{\delta}}+\hat{v}_{1}^{*} \frac{\partial v_{1}^{*}}{\partial x_{1}^{*}}+\hat{v}_{2}^{*} \frac{\partial v_{2}^{*}}{\partial x_{1}^{*}} \frac{\hat{\delta}^{2}}{L^{2}} \\
& +\frac{\hat{P}}{V_{1} \hat{V}_{1}} \frac{\partial \hat{p}^{*}}{\partial x_{1}^{*}}-\frac{1}{\operatorname{Re}_{\mathrm{L}}}\left[\frac{\partial^{2} \hat{v}_{1}^{*}}{\partial x_{1}^{* 2}}+\frac{\partial^{2} \hat{v}_{1}^{*}}{\partial x_{2}^{* 2}} \frac{L^{2}}{\hat{\delta}^{2}}\right] \\
& =-\frac{\partial j_{\Omega}^{*}}{\partial v_{1}^{*}} \frac{J_{\Omega} L}{V_{1}^{2} \hat{V}_{1}} \\
& \mathrm{R}_{2}^{\hat{v} *}:-v_{1}^{*} \frac{\partial \hat{v}_{2}^{*}}{\partial x_{1}^{*}}-v_{2}^{*} \frac{\partial \hat{v}_{2}^{*}}{\partial x_{2}^{*}} \frac{\delta}{\hat{\delta}}+\hat{v}_{1}^{*} \frac{\partial v_{1}^{*}}{\partial x_{2}^{*}} \frac{L^{2}}{\delta \hat{\delta}}+\hat{v}_{2}^{*} \frac{\partial v_{2}^{*}}{\partial x_{2}^{*}} \\
& +\frac{\partial \hat{p}^{*}}{\partial x_{2}^{*}} \frac{\hat{P}}{\hat{V}_{1} V_{1}} \frac{L^{2}}{\hat{\delta}^{2}}-\frac{1}{\operatorname{Re}_{\mathrm{L}}}\left[\frac{\partial^{2} \hat{v}_{2}^{*}}{\partial x_{1}^{* 2}}+\frac{L^{2}}{\hat{\delta}^{2}} \frac{\partial^{2} \hat{v}_{2}^{*}}{\partial x_{2}^{* 2}}\right] \\
& =-\frac{\partial j_{\Omega}^{*}}{\partial v_{2}^{*}} \frac{J_{\Omega} L^{3}}{V_{1}^{2} \hat{V}_{1} \delta \hat{\delta}}, \\
& \mathrm{R}^{\hat{p}^{*}}:-\frac{\partial \hat{v}_{1}^{*}}{\partial x_{1}^{*}}-\frac{\partial \hat{v}_{2}^{*}}{\partial x_{2}^{*}}=0,
\end{aligned}
$$

by assuming $\hat{V}_{2} \propto \hat{V}_{1} \hat{\delta} / L$.

\section{APPENDIX C: PRIMAL AND ADJOINT SIMILARITY RELATIONS}

The relations in Table III simplify the tangential primal and the tangential and normal adjoint BL equations. 
TABLE III. Similarity relations for the primal and adjoint boundary-layer equations.

\begin{tabular}{lcccc}
\hline \hline Primal quantity/operator & $v_{1}$ & $v_{2}$ & $\partial v_{1} / \partial x_{1}$ & $\partial v_{1} / \partial x_{2}$ \\
Similarity transformation & $V_{1} f^{\prime}$ & $\frac{1}{2} \sqrt{\frac{\nu b^{2} V_{1}}{a+b x_{1}}}\left[f^{\prime} \eta-f\right]$ & $-\frac{1}{2} \frac{V_{1} b}{a+b x_{1}} f^{\prime \prime} \eta$ & $V_{1} \sqrt{\frac{V_{1}}{\nu\left(a+b x_{1}\right)} f^{\prime \prime}} V_{1} \frac{V_{1}}{\nu\left(a+b x_{1}\right)} f^{\prime \prime \prime}$ \\
Adjoint quantity/operator & $\hat{v}_{1}$ & $\hat{v}_{2}$ & $\partial \hat{v}_{1} / \partial x_{1}$ & $\partial \hat{v}_{1} / \partial x_{2}$ \\
Similarity transformation & $\hat{V}_{1} \hat{f}^{\prime}$ & $\frac{1}{2} \sqrt{\frac{\nu b^{2} V_{1}}{a+b x_{1}}\left[\hat{f}^{\prime} \hat{\eta}-\hat{f}\right]}$ & $-\frac{1}{2} \frac{\hat{V}_{1} b}{a+b x_{1}} \hat{f}^{\prime \prime} \hat{\eta}$ & $\hat{V}_{1} \sqrt{\frac{V_{1}}{\nu\left(a+b x_{1}\right)} \hat{f}^{\prime \prime}} \hat{V}_{1} \frac{\partial^{2} \hat{v}_{1} / \partial x_{2}^{2}}{\nu\left(a+b x_{1}\right)} \hat{f}^{\prime \prime \prime}$ \\
\hline \hline
\end{tabular}

\section{DATA AVAILABILITY}

The data that support the findings of this study are available from the corresponding author upon reasonable request.

\section{REFERENCES}

${ }^{1}$ C. Airiau, "Non-parallel acoustic receptivity of a Blasius boundary layer using an adjoint approach," Flow, Turbul. Combust. 65(3-4), 347-367 (2000).

${ }^{2}$ C. Airiau, A. Bottaro, S. Walther, and D. Legendre, "A methodology for optimal laminar flow control: Application to the damping of TollmienSchlichting waves in a boundary layer," Phys. Fluids 15(5), 1131-1145 (2003).

${ }^{3}$ C. Airiau, S. Walther, and A. Bottaro, "Boundary layer sensitivity and receptivity,” C. R. Méc. 330(4), 259-265 (2002).

${ }^{4}$ W. K. Anderson and D. L. Bonhaus, "Airfoil design on unstructured grids for turbulent flows,” AIAA J. 37(2), 185-191 (1999).

${ }^{5}$ H. Blasius, Grenzschichten in Flüssigkeiten Mit Kleiner Reibung (Druck von BG Teubner, 1907).

${ }^{6}$ A. Bottaro, P. Corbett, and P. Luchini, "The effect of base flow variation on flow stability," J. Fluid Mech. 476, 293 (2003).

${ }^{7}$ J. Elliott and J. Peraire, "Practical 3D aerodynamic design and optimization using unstructured meshes," AIAA J. 35(9), 1479-1485 (1997).

${ }^{8}$ F. Giannetti, S. Camarri, and P. Luchini, "Structural sensitivity of the secondary instability in the wake of a circular cylinder," J. Fluid Mech. 651, 319 (2010).

${ }^{9}$ F. Giannetti and P. Luchini, "Leading-edge receptivity by adjoint methods," J. Fluid Mech. 547(21), 21 (2006).

${ }^{10} \mathrm{~F}$. Giannetti and P. Luchini, "Structural sensitivity of the first instability of the cylinder wake,” J. Fluid Mech. 581(1), 167-197 (2007).

${ }^{1}$ M. B. Giles and N. A. Pierce, "Adjoint equations in CFD: Duality, boundary conditions and solution behaviour,” AIAA Paper No. AIAA-97, 1850.

${ }^{12}$ M. B. Giles and N. A. Pierce, "An introduction to the adjoint approach to design,” Flow, Turbul. Combust. 65(3), 393-415 (2000).

${ }^{13}$ A. Griewank, "On automatic differentiation," in Mathematical Programming, Applications (Kluwer Academic Publishers, 1989), pp. 83-108.

${ }^{14} \mathrm{~A}$. Griewank and A. Walther, "Revolve-reverse or adjoint mode of computational differentiation," Trans. Math. Softw. 26(1), 19-45 (2000).

${ }^{15}$ T. Herbert, "Parabolized stability equations," Annu. Rev. Fluid Mech. 29(1), 245-283 (1997).

${ }^{16}$ D. C. Hill, "A theoretical approach for analyzing the restabilization of wakes," in 30th Aerospace Sciences Meeting and Exhibit (1992), p. 67.

${ }^{17}$ D. C. Hill, "Adjoint systems and their role in the receptivity problem for boundary layers," J. Fluid Mech. 292, 183-204 (1995).

${ }^{18} \mathrm{Y}$. Hwang and H. Choi, "Control of absolute instability by basic-flow modification in a parallel wake at low Reynolds number," J. Fluid Mech. 560, 465 (2006).

${ }^{19}$ C. S. Kapellos, E. M. Papoutsis-Kiachagias, K. C. Giannakoglou, and M. Hartmann, "The unsteady continuous adjoint method for minimizing flowinduced sound radiation," J. Comput. Phys. 392, 368-384 (2019).
${ }^{20}$ G. K. Karpouzas, E. M. Papoutsis-Kiachagias, T. Schumacher, E. Villiers, K. C. Giannakoglou, and C. Othmer, "Adjoint optimization for vehicle external aerodynamics,” Int. J. Automot. Eng. 7(1), 1-7 (2016).

${ }^{21}$ J. Kröger, N. Kühl, and T. Rung, "Adjoint volume-of-fluid approaches for the hydrodynamic optimisation of ships,” Ship Technol. Res. 65(1), 47-68 (2018).

${ }^{22}$ J. Kröger and T. Rung, "CAD-free hydrodynamic optimisation using consistent Kernel-based sensitivity filtering," Ship Technol. Res. 62(3), 111-130 (2015).

${ }^{23}$ N. Kühl, Numerical Approximation of the Adjoint Blasius Equation (MATLAB Central File Exchange, 2020).

${ }^{24}$ N. Kühl, J. Kröger, M. Siebenborn, M. Hinze, and T. Rung, "Adjoint complement to the volume-of-fluid method for immiscible flows," preprint arXiv:2009.03957 (2020).

${ }^{25}$ N. Kühl, P. M. Müller, A. Stück, M. Hinze, and T. Rung, "Decoupling of control and force objective in adjoint-based fluid dynamic shape optimization," AIAA J. 57(9), 4110-4114 (2019).

${ }^{26} \mathrm{P}$. Luchini and A. Bottaro, "Adjoint equations in stability analysis," Annu. Rev. Fluid Mech. 46, 493 (2014).

${ }^{27}$ S. K. Nadarajah, "The discrete adjoint approach to aerodynamic shape optimization," Ph.D. thesis (Stanford University, 2003).

${ }^{28}$ S. K. Nadarajah and A. Jameson, "A comparison of the continuous and discrete adjoint approach to automatic aerodynamic optimization," in 38th Aerospace Sciences Meeting and Exhibit (2000), p. 667.

${ }^{29} \mathrm{C}$. Othmer, "A continuous adjoint formulation for the computation of topological and surface sensitivities of ducted flows," Int. J. Numer. Methods Fluids 58(8), 861-877 (2008).

${ }^{30}$ C. Othmer, “Adjoint methods for car aerodynamics," J. Math. Ind. 4(1), 6 (2014).

${ }^{31}$ E. M. Papoutsis-Kiachagias and K. C. Giannakoglou, "Continuous adjoint methods for turbulent flows, applied to shape and topology optimization: Industrial applications,” Arch. Comput. Methods Eng. 23(2), 255 (2016).

${ }^{32}$ J. E. V. Peter and R. P. Dwight, "Numerical sensitivity analysis for aerodynamic optimization: A survey of approaches," Comput. Fluids 39(3), 373-391 (2010).

${ }^{33}$ J. O. Pralits, A. Hanifi, and D. S. Henningson, "Adjoint-based optimization of steady suction for disturbance control in incompressible flows," J. Fluid Mech. 467, 129 (2002).

${ }^{34}$ T. Rung, K. Wöckner, M. Manzke, J. Brunswig, C. Ulrich, and A. Stück, "Challenges and perspectives for maritime CFD applications," Jahrb. Schiffbautech. Ges. 103, 127-139 (2009).

${ }^{35} \mathrm{H}$. Schlichting and K. Gersten, Grenzschicht-Theorie (Springer-Verlag, 2006).

${ }^{36}$ L.-U. Schrader, L. Brandt, and T. A. Zaki, "Receptivity, instability and breakdown of Görtler flow,” J. Fluid Mech. 682, 362-396 (2011).

${ }^{37} \mathrm{~V}$. Schulz and M. Siebenborn, "Computational comparison of surface metrics for PDE constrained shape optimization," Comput. Methods Appl. Math. 16(3), 485-496 (2016).

${ }^{38} \mathrm{O}$. Soto and R. Löhner, "On the computation of flow sensitivities from boundary integrals," in 42nd AIAA Aerospace Sciences Meeting and Exhibit (2004).

${ }^{39} \mathrm{~J}$. Springer and K. Urban, "Adjoint-based optimization for rigid body motion in multiphase Navier-Stokes flow,” SIAM J. Sci. Comput. 37(2), B185-B214 (2015).

${ }^{40} \mathrm{~A}$. Stück and T. Rung, "Adjoint complement to viscous finite-volume pressurecorrection methods,” J. Comput. Phys. 248, 402-419 (2013).

${ }^{41}$ D. Tempelmann, A. Hanifi, and D. S. Henningson, "Swept-wing boundarylayer receptivity,” J. Fluid Mech. 700, 490-501 (2012). 
${ }^{42} \mathrm{~J}$. Vassberg and A. Jameson, "Aerodynamic shape optimization part 2: Sample applications," in Introduction to Optimization and Multidisciplinary Design (Von Karman Institute for Fluid Dynamics Brussels, 2006), pp. 1-41.

${ }^{43} \mathrm{~J}$. Vassberg and A. Jameson, "Aerodynamic shape optimization part I: Theoretical background," in Introduction to Optimization and Multidisciplinary Design (Von Karman Institute for Fluid Dynamics Brussels, 2006), pp. 1-30.
${ }^{44}$ S. Yakubov, B. Cankurt, M. Abdel-Maksoud, and T. Rung, "Hybrid MPI/ OpenMP parallelization of an Euler-Lagrange approach to cavitation modelling," Comput. Fluids 80, 365-371 (2013).

${ }^{45}$ S. Yakubov, T. Maquil, and T. Rung, "Experience using pressure-based CFD methods for Euler-Euler simulations of cavitating flows," Comput. Fluids 111, 91-104 (2015). 